\title{
Hilbert transform, Toeplitz operators and Hankel operators, and invariant $A_{\infty}$ weights
}

\section{Sergei Treil, Alexander Volberg and Dechao Zheng}

Abstract. In this paper, several sufficient conditions for boundedness of the Hilbert transform between two weighted $L^{p}$-spaces are obtained. Invariant $A_{\infty}$ weights are introduced. Several characterizations of invariant $A_{\infty}$ weights are given. We also obtain some sufficient conditions for products of two Toeplitz operators or Hankel operators to be bounded on the Hardy space of the unit circle using Orlicz spaces and Lorentz spaces.

\section{Introduction.}

Let $\partial D$ be the unit circle and $d w$ denotes the Lebesgue measure on $\partial D$. For $p>1$ and $v$ a positive function on $\partial D, L^{p}(v)$ denotes the space of functions $f$ on the unit circle such that

$$
\int_{\partial D}|f(w)|^{p} v(w) d w<\infty
$$

We use $L^{p}$ to denote $L^{p}(v)$ if $v=1$. Let $H^{p}$ be the subspace of $L^{p}$ which those functions are analytic on the unit disk $D$. There is an orthogonal projection $P$ from $L^{2}$ onto $H^{2}$. The Hilbert transform $T$ is defined to be $T=-i P+i(I-P)$. 
We are concerned with the problem of identifying those pair $(v, u)$ of positive functions on $\partial D$ for which the Hilbert transform $T$ is bounded from $L^{p}(u)$ to $L^{p}(v)$, that is

$$
\int_{\partial D}|T f(w)|^{p} v(w) d w \leq C \int_{\partial D}|f(w)|^{p} u(w) d w
$$

for all $f \in L^{p}(u)$.

This problem was first raised by Muckenhoupt and Wheeden in [16]. There is a very elegant theorem of Cotlar-Sadosky ([4], [5]) which gives a necessary and sufficient condition that (1) holds for a given constant $C$. The theorem of Cotlar and Sadosky generalizes the HelsonSzegö theorem in two weights case. On the other hand, it is a considerably interesting question in harmonic analysis to find explicit estimates of the norm of the Hilbert transform between two weighted spaces; see [2] for further references. So it seems interesting to find characterizations of the weight functions for (1) close in form to the following $A_{p}$ condition [15]. It remains an open question ([6], [22], and [8]).

In case that $u=v$, Hunt, Muckenhoupt and Wheeden [12] have proved that (1) holds if and only if $v$ satisfies a simpler condition

$$
\left(A_{p}\right) \quad \sup _{I}\left(\frac{1}{|I|} \int_{I} v(w) d w\right)\left(\frac{1}{|I|} \int_{I} v(w)^{-1 /(p-1)} d w\right)^{p-1}<\infty
$$

where the supremum is taken over all arcs $I$. Muckenhoupt [14] has shown that the $A_{p}$ condition is a necessary and sufficient condition that the Hardy-Littlewood maximal function

$$
M f(x)=\sup _{x \in I} \frac{1}{|I|} \int_{I}|f(w)| d w
$$

satisfies the following inequality

$$
\int_{\partial D}|M f(w)|^{p} v(w) d w \leq C \int_{\partial D}|f(w)|^{p} u(w) d w
$$

One may expect that the following condition is a necessary and sufficient condition that inequality (1) holds even $u \neq v$

$$
\left(A_{p}^{\prime}\right) \quad \sup _{I}\left(\frac{1}{|I|} \int_{I} v(w) d w\right)\left(\frac{1}{|I|} \int_{I} u(w)^{-1 /(p-1)} d w\right)^{p-1}<\infty .
$$


Simple examples [16] show that the $A_{p}^{\prime}$ condition is not sufficient for either (1) or (2) to hold. Sawyer [21] has shown that (2) holds if and only if

$$
\begin{gathered}
\sup _{I}\left(\int_{I}\left(M\left(\chi_{I} u^{-1 /(1-p)}\right)(w)\right)^{p} v(w) d w\right) \\
\cdot\left(\int_{I} u(w)^{-1 /(p-1)} d w\right)^{-1}<\infty,
\end{gathered}
$$

where $\chi_{I}$ denotes the characteristic function of $I$. Sawyer [22] also showed that the $S_{p}$ condition with additional conditions is sufficient for (1). Fujii [8] has obtained the following sufficient condition for (1):

There exist constants $0<\alpha<1, \beta$ and $0<C_{0}<\infty$ such that, for every arc $I$ and all measurable subsets $E$ and $F$ of $I$ with $E \cap F=0$ and $|F| \geq \alpha|I|$,

$$
\begin{aligned}
\left(\int_{E} v(w) d w\right)\left(|I|^{-1}\right. & \left.\int_{c(n, \alpha) I} u^{-1 /(p-1)}(w) d w\right)^{p} \\
& \leq C_{0}\left(\frac{|E|}{|I|}\right)^{\beta} \int_{F} u^{-1 /(p-1)}(w) d w \\
& <\infty,
\end{aligned}
$$

where $c(n, \alpha)$ is a constant greater than 1 and $c(n, \alpha) I$ is the arc with the same center as $I$ and expanded $c(n, \alpha)$ times.

Sawyer's condition involves the operator $M$, and it is interesting to obtain sufficient conditions close in form to the $A_{p}^{\prime}$ condition. In that direction, Neugebauer [17] has obtained the following sufficient condition for (2) for $r>1$,

$$
\sup _{I}\left(\frac{1}{|I|} \int_{I} v^{r}(x) d x\right)\left(\frac{1}{|I|} \int_{I} u^{-r /(p-1)}(x) d x\right)^{p-1}<\infty
$$

Recently Pérez [19] has improved the condition (3) and obtained weaker sufficient conditions for (2) using the general maximal operator involving in Banach function spaces.

In this paper, using Banach function norms we define a maximal operator and a nontangential maximal operator. We shall show several 
sufficient conditions for (1). In particular, we prove that if $(u, v)$ is a pair of weights such that for some $r>1$,

$$
\sup _{z \in D} v^{r}(z)\left(u^{-r /(p-1)}(z)\right)^{p-1}<\infty,
$$

then the Hilbert transform is bounded from $L^{p}(u)$ to $L^{p}(v)$. Here we follow the convention of identifying functions on the unit circle with their harmonic extensions, defined via Poisson's formula, into the unit disk $D$.

The condition (4) is analogous to the condition (3) and the following condition: for $0<\alpha<1$ and $r>1$,

$$
\begin{aligned}
\sup _{I}|I|^{\alpha / n}\left(\frac{1}{|I|}\right. & \left.\int_{I} v^{r}(x) d x\right)^{1 /(p r)} \\
& \cdot\left(\frac{1}{|I|} \int_{I} u^{\left(1-p^{\prime}\right) r}(x) d x\right)^{1 /\left(p^{\prime} r\right)}<\infty .
\end{aligned}
$$

Sawyer and Wheeden [23] have shown that the condition (5) is a sufficient condition for fractional integral operators

$$
I_{\alpha} f(x)=\int|x-y|^{\alpha-1} f(y) d y
$$

to be bounded from $L^{p}(u)$ to $L^{p}(v)$.

For $z \in D$, let $\phi_{z}(w)$ be the Möbius map

$$
\phi_{z}(w)=\frac{z-w}{1-\bar{z} w},
$$

for $w \in \bar{D}$.

We can improve the condition (4) using the scale of Lorentz spaces or Orlicz spaces which are concrete examples of Banach function spaces. Let $P(z, x)$ be the Poisson kernel

$$
P(z, x)=\frac{1-|z|^{2}}{|1-\bar{z} x|^{2}} .
$$

We shall show that one of the following conditions is a sufficient condition for (1)

$$
\begin{aligned}
\sup _{z \in D}\left(\sup _{t>0}\right. & \left.\int_{v(x)>t} t^{r} P(z, x) d x\right) \\
& \cdot\left(\sup _{t>0}\left(\int_{u^{-1}(x)>t} t^{r /(p-1)} P(z, x) d x\right)^{p-1}\right)<\infty,
\end{aligned}
$$


for some $r>1$, and

$$
\sup _{z \in D}\left\|v^{1 / p} \circ \phi_{z}\right\|_{L^{\Phi}}\left\|u^{-1} \circ \phi_{z}\right\|_{L^{\Psi}}<\infty
$$

for some Young functions such as

$$
\Phi(t)=t^{p} \log ^{p-1+\delta}(1+t)
$$

and

$$
\Psi(t)=t^{p^{\prime}} \log p^{p^{\prime}-1+\delta}(1+t)
$$

or weaker ones

$$
\Phi(t)=t^{p} \log ^{p-1}(1+t)(\log \log (1+t))^{p-1+\delta}
$$

and

$$
\Psi(t)=t^{p^{\prime}} \log p^{p^{\prime}-1}(1+t)(\log \log (1+t))^{p^{\prime}-1+\delta},
$$

for some $\delta>0$.

We will introduce invariant $A_{\infty}$ weights and give several characterizations of invariant $A_{\infty}$ weights. From these characterizations we can easily tell the difference between $A_{\infty}$ and invariant $A_{\infty}$ weights.

If we assume that both $v$ and $u^{-1 /(p-1)}$ are invariant $A_{\infty}$ weights, we will show that the condition

$$
\sup _{z \in D} v(z)\left(u^{-1 /(p-1)}(z)\right)^{p-1}<\infty
$$

is a necessary and sufficient condition for the Hilbert transform to be uniformly bounded from $L^{p}\left(u \circ \phi_{z}\right)$ to $L^{p}\left(v \circ \phi_{z}\right)$ for all $z \in D$.

Boundedness of the Hilbert transform between two weighted $L^{2}$ spaces is related to boundedness of products of two Toeplitz operators or Hankel operators on the Hardy space $H^{2}$. On products of Toeplitz operators Sarason [20] made the following conjecture:

Let $f$ and $g$ be outer functions in $H^{2}$. The product $T_{f} T_{\bar{g}}$ is bounded if and only if

$$
\sup _{z \in D}|f|^{2}(z)|g|^{2}(z)<\infty
$$


On the product of Hankel operators it is natural to make the following conjecture [27]: only if

Let $f$ and $g$ be in $L^{2}$. Then the product $H_{f}^{*} H_{g}$ is bounded if and

$$
\sup _{z \in D}\left\|H_{f} k_{z}\right\|_{2}\left\|H_{g} k_{z}\right\|_{2}<\infty
$$

Let $f_{-}$denote $(1-P) f$ for $f \in L^{2}$. Then the condition (10) is equivalent to

$$
\sup _{z \in D}\left\|f_{-} \circ \phi_{z}-f_{-}(z)\right\|_{2}\left\|g_{-} \circ \phi_{z}-g_{-}(z)\right\|_{2}<\infty
$$

Treil [20] showed that if the product $T_{f} T_{\bar{g}}$ is bounded, then the condition (9) holds in Sarason's Conjecture. Conversely, it was shown [27] that the following condition implies that $T_{f} T_{\bar{g}}$ is bounded

$$
\sup _{z \in D}|f|^{2 r}(z)|g|^{2 r}(z)<\infty
$$

for some $r>1$. Also in [27] it was shown that the condition (10) is necessary for $H_{f}^{*} H_{g}$ to be bounded and that the following condition is sufficient

$$
\sup _{z \in D}\left\|f_{-} \circ \phi_{z}-f_{-}(z)\right\|_{2 r}\left\|g_{-} \circ \phi_{z}-g_{-}(z)\right\|_{2 r}<\infty
$$

for some $r>1$.

In this paper we will improve the above sufficient conditions for boundedness of the product of two either Toeplitz operators or Hankel operators using Orlicz spaces or Lorentz spaces. In particular, we will show that if for two outer functions $f$ and $g$ in $H^{2}$,

$$
\sup _{z \text { inD }}\left\|f \circ \phi_{z}\right\|_{L^{\Phi}}\left\|g \circ \phi_{z}\right\|_{L^{\Phi}}<\infty
$$

then $T_{f} T_{\bar{g}}$ is bounded, and if for two functions $f$ and $g$ in $L^{2}$,

$$
\sup _{z \in D}\left\|f_{-} \circ \phi_{z}-f_{-}(z)\right\|_{L^{\Phi}}\left\|g_{-} \circ \phi_{z}-g_{-}(z)\right\|_{L^{\Phi}}<\infty,
$$


then $H_{f}^{*} H_{g}$ is bounded. Here the Young function $\Phi$ is $t^{2} \log ^{1+\delta}(1+t)$ or $t^{2} \log (1+t)(\log \log (1+t))^{1+\delta}$ for some $\delta>0$.

The letter $C$ will denote a positive constant, possibly different on each occurrence.

\section{Banach function spaces.}

Recall some basic facts about the theory of Banach function spaces, Orlicz spaces and Lorentz spaces. We shall refer the reader to [1], [13] and [18] for a complete account. A Banach function space $X$ over the unit circle is a subspace of the Lebesgue measurable functions with a Banach function norm. The most important property of the Banach function space is the generalized Hölder inequality

$$
\int|f(x) g(x)| d x \leq\|f\|_{X}\|g\|_{X^{\prime}}
$$

where $X^{\prime}$ is the associate space to $X$.

Let us look at several concrete examples of Banach function spaces. A function $\Phi:[0, \infty) \rightarrow[0, \infty)$ is a Young function if it is continuous, convex and increasing satisfying $B(0)=0$ and $B(t) \rightarrow \infty$ as $t \rightarrow \infty$. Each Young function $\Phi$ has associated a complementary Young function $\tilde{\Phi}$. The Orlicz space $L^{\Phi}$ consists of all Lebesgue measurable functions $f$ such that

$$
\int \Phi\left(\frac{|f(x)|}{\lambda}\right) d x<\infty
$$

for some $\lambda>0$. The space $L^{\Phi}$ is a Banach function space with the Luxemburg norm defined by

$$
\|f\|_{\Phi}=\inf \left\{\lambda>0: \int \Phi\left(\frac{|f(x)|}{\lambda}\right) d x \leq 1\right\}
$$

Its associated space is $L^{\tilde{\Phi}}$. A Young function $\Phi$ is said to satisfy the $\Delta_{2}$-condition if there exist $C>0$ and $T \geq 0$ such that

$$
\Phi(2 t) \leq C \Phi(t),
$$

for all $t \geq T$. 
Let $1<s, q \leq \infty$. The Lorentz space $L^{s, q}$ is the space of the Lebegue measurable functions $f$ such that

$$
\|f\|_{L^{s, q}}=\left(q \int_{0}^{\infty}\left(t|\{x \in \partial D:|f(x)|>t\}|^{1 / s}\right)^{q} \frac{d t}{t}\right)^{1 / q}<\infty,
$$

if $q<\infty$, and

$$
\|f\|_{L^{s, \infty}}=\sup _{0<t<\infty} t|\{x \in \partial D:|f(x)|>t\}|^{1 / s}<\infty,
$$

if $q=\infty$. The Lorentz space $L^{s, q}$ is a Banach function space with the associate space $L^{s^{\prime}, q^{\prime}}$.

Let $X$ be a Banach function space over $\partial D$ with respect to the Lebesgue measure. Given a measurable function $f$ and any interval $I$ we define the $X$-average of $f$ over $I$ by

$$
\|f\|_{X, I}=\left\|\tau_{|I|}\left(f \chi_{I}\right)\right\|_{X}
$$

where $\tau_{\delta}$, with $\delta>0$, is the dilation operator $\tau_{\delta} f(x)=f(\delta x), \chi_{E}$ is the characteristic function of $E$. We define a natural maximal operator $M_{X} f(x)$ associated to the space $X$ by

$$
M_{X} f(x)=\sup _{x \in I}\|f\|_{X, I}
$$

where the supremum is taken over all intervals containing $x$.

For any $x \in \partial D$, and a fixed $\alpha>1$ let

$$
\Gamma(x)=\left\{z \in D: \frac{|x-z|}{1-|z|} \leq \alpha\right\}
$$

We define a nontangential maximal operator $N_{X} f(x)$ associated to the space $X$ by

$$
N_{X} f(x)=\sup _{z \in \Gamma(x)}\left\|f \circ \phi_{z}\right\|_{X}
$$

Let $X=L^{\Phi}$ be the Orlicz space defined by a Young function $\Phi$. Then the maximal operator $M_{X}$ is defined in terms of the average

$$
\|f\|_{X, I}=\inf \left\{\lambda>0: \frac{1}{|I|} \int_{I} \Phi\left(\frac{|f(x)|}{\lambda}\right) d x \leq 1\right\}
$$


If $X$ is the Lorentz space $X=L^{s, q}$, then the maximal operator is

$$
M_{X} f(x)=\sup _{x \in I} \frac{1}{|I|^{1 / s}}\left\|f \chi_{I}\right\|_{L^{s, q}}
$$

Proposition 1. Let $X$ be either an Orlicz space or a Lorentz space. Let $f$ be in $X$. Then there is a constant $C$ such that

$$
N_{X} f(x) \leq C M_{X} f(x)
$$

for $x \in \partial D$.

Proof. We shall consider only the case that $X$ is the Orlicz space $L^{\Phi}$ with a Young function $\Phi$. The same method will prove the theorem in the case that $X$ is a Lorentz space.

We may assume $x=1$. We will use polar coordinate $r e^{i t}$ for points in the unit disk. Let us first consider the points $z=r$ on the real axis in the cone

$$
\Gamma(1)=\left\{z \in D: \frac{|1-z|}{1-|z|}<\alpha\right\}
$$

Then

$$
f(r)=\int P\left(r, e^{i t}\right) f\left(e^{i t}\right) d t
$$

and the kernel $P\left(r, e^{i t}\right)$ is a positive even function which is decreasing for positive $t$. That means $P\left(r, e^{i t}\right)$ is a convex combination of the box kernels $\chi_{(-h, h)}(t) /(2 h)$. Take step functions $h_{n}(t)$, which are also nonnegative, even, and decreasing on $t>0$, such that $h_{n}(t)$ increases with $n$ to $P\left(r, e^{i t}\right)$. Then $h_{n}(t)$ has the form

$$
\sum_{j=1}^{N} a_{j} \chi_{\left(-t_{j}, t_{j}\right)}(t)
$$

with $a_{j} \geq 0$, and $\int h_{n}(t) d x=\sum_{j} 2 t_{j} a_{j} \leq 1$. Suppose that $\lambda>0$ such that $\lambda>M_{X} f(1)$. Thus

$$
\left(\frac{1}{|I|}\right) \int_{I} \Phi\left(\frac{\left|f\left(e^{i t}\right)\right|}{\lambda}\right) d t \leq 1
$$


for all intervals $I$ containing 1 . Then

$$
\begin{aligned}
\left|\int h_{n}(t) \Phi\left(\frac{\left|f\left(e^{i t}\right)\right|}{\lambda}\right) d t\right| & \leq \sum_{j=1}^{N} 2 t_{j} a_{j} \frac{1}{\left|\left(-t_{j}, t_{j}\right)\right|} \int_{\left(-t_{j}, t_{j}\right)} \Phi\left(\frac{\left|f\left(e^{i t}\right)\right|}{\lambda}\right) d t \\
& \leq \sum_{j=1}^{n} 2 t_{j} a_{j} \\
& \leq 1
\end{aligned}
$$

Then by monotone convergence

$$
\int \Phi\left(\frac{\left|f \circ \phi_{r}\left(e^{i t}\right)\right|}{\lambda}\right) d t=\int \Phi\left(\frac{\left|f\left(e^{i t}\right)\right|}{\lambda}\right) P\left(r, e^{i t}\right) d t \leq 1 .
$$

Hence $\left\|f \circ \phi_{r}\right\|_{X} \leq \lambda$. Now fix $z \in \Gamma(1)$. Then $\left|1-r e^{i s}\right| \leq \alpha(1-r)$, and $P\left(r e^{i s}, e^{i t}\right)$ is majorized by a positive even function $\psi(t)$, which is decreasing on $t>0$, such that

$$
\int \psi(t) d t \leq A_{\alpha}
$$

for some constant $A_{\alpha}$. The function is $\psi(t)=\sup \left\{P\left(r e^{i s}, e^{i l}\right):|l|>t\right\}$. Approximating $\psi(t)$ from below by step functions $h_{n}(t)$ just as before, we have

$$
\int \psi(t) \Phi\left(\frac{\left|f\left(e^{i t}\right)\right|}{\lambda}\right) d t \leq A_{\alpha}
$$

By convexity, we obtain

$$
\begin{aligned}
\int \Phi\left(\frac{\left|f \circ \phi_{z}\left(e^{i t}\right)\right|}{A_{\alpha} \lambda}\right) d t & =\int \Phi\left(\frac{\left|f\left(e^{i t}\right)\right|}{A_{\alpha} \lambda}\right) P\left(z, e^{i t}\right) d t \\
& \leq \int \Phi\left(\frac{\left|f\left(e^{i t}\right)\right|}{A_{\alpha} \lambda}\right) \psi(t) d t \\
& \leq 1 .
\end{aligned}
$$

Thus by the definition of the Luxemburg norm, we have

$$
\left\|f \circ \phi_{z}\right\|_{X}<A_{\alpha} \lambda
$$

Therefore

$$
N_{X} f(1) \leq A_{\alpha} \lambda
$$


So we conclude that

$$
N_{X} f(1) \leq A_{\alpha} M_{X} f(1)
$$

\section{Distribution function inequality.}

In this section we will get two distribution function inequalities involving the Lusin area integral and the nontangential maximal operator $N_{X}$ for Banach function spaces $X$.

For $w$ a point in $\partial D$, we let $\Gamma_{w}$ denote the angle with vertex $w$ and opening $\pi / 2$ which is bisected by the radius to $w$. The set of points $z$ in $\Gamma_{w}$ satisfying $|z-w|<\varepsilon$ will be denoted by $\Gamma_{w, \varepsilon}$. We fix the shape of our typical truncated cone $\Gamma_{w, \varepsilon}$. Whenever $h$ is in $L^{1}$, we define the truncated Lusin area integral of $h$ to be

$$
A_{\varepsilon}(h)(w)=\left(\int_{\Gamma_{w, \varepsilon}}|\nabla h(z)|^{2} d A(z)\right)^{1 / 2} .
$$

Here $h(z)$ means the harmonic extension of $h$ on $D$,

$$
|\nabla h(z)|^{2}=\left|\frac{\partial h}{\partial z}\right|^{2}+\left|\frac{\partial h}{\partial \bar{z}}\right|^{2}
$$

and $d A(z)$ denotes the area measure on the unit disk. Then $A_{\varepsilon}(h)(w)^{2}$ represents the area (points counted with their multiplicity) of the image in the complex plane of the truncated cone $\Gamma_{w, \varepsilon}$ under the map $z \rightarrow$ $h(z)$.

The Lebesgue measure of the subset $E$ of $\partial D$ will be denoted by $|E|$. For $z \in D$, we let $I_{z}$ denote the closed subarc of $\partial D$ with center $z /|z|$ and measure $\delta(z)=1-|z|$.

For a number $p>1$, we use $p^{\prime}$ to denote the number so that $1 / p+1 / p^{\prime}=1$. Let $Q$ denote the operator $I-P$. As in [27], we have the following distribution function inequalities.

Theorem 1. Let $X$ and $Y$ be two Banach function spaces. Let $f$ and $g$ be in $X$ and $Y$ respectively, and $\phi$ and $\psi$ in $X^{\prime}$ and $Y^{\prime}$. For $|z|>1 / 2$ and $a>0$ sufficiently large, there is a constant $C_{a}>0$ such that

$$
\begin{aligned}
\mid\left\{\lambda \in I_{z}:\right. & A_{2 \delta(z)}(P(f \phi))(\lambda) A_{2 \delta(z)}(P(g \psi))(\lambda) \\
< & a\left\|f \circ \phi_{z}\right\|_{X}\left\|g \circ \phi_{z}\right\|_{Y} \\
& \left.\quad \inf _{w \in I_{z}} N_{X^{\prime}}(\phi)(w) \inf _{w \in I_{z}} N_{Y^{\prime}}(\psi)(w)\right\}\left|\geq C_{a}\right| I_{z} \mid
\end{aligned}
$$


and

$$
\begin{aligned}
\mid\left\{\lambda \in I_{z}:\right. & A_{2 \delta(z)}(Q(f \phi))(\lambda) A_{2 \delta(z)}(Q(g \psi))(\lambda) \\
< & a\left\|f_{-} \circ \phi_{z}-f_{-}(z)\right\| X\left\|g_{-} \circ \phi_{z}-g_{-}(z)\right\|_{Y} \\
& \left.\quad \inf _{w \in I_{z}} N_{X^{\prime}}(\phi)(w) \inf _{w \in I_{z}} N_{Y^{\prime}}(\psi)(w)\right\}\left|\geq C_{a}\right| I_{z} \mid .
\end{aligned}
$$

Moreover, the constant $C_{a}$ can be chosen to satisfy $C_{a}=1-2 a^{-1 / 2} C$ for some positive constant $C$.

Proof. We will show only the first distribution function inequality. The same method will prove the second one.

For a fixed $z$ in $D$, and $a>0$ let $E(a)$ be the set of points in $I_{z}$ where

$$
A_{2 \delta(z)}(P(f \phi))(\lambda) \leq a^{1 / 2}\left\|f \circ \phi_{z}\right\|_{X} \inf _{w \in I_{z}} N_{X^{\prime}} \phi(w)
$$

and $F(a)$ the set of points in $I_{z}$ where

$$
A_{2 \delta(z)}(P(g \psi)(\lambda)) \leq a^{1 / 2}\left\|g \circ \phi_{z}\right\|_{Y} \inf _{w \in I_{z}} N_{Y^{\prime}} \psi(w) .
$$

We claim the following distribution function inequalities, i.e. for $a>0$ sufficiently large

$$
|E(a)| \geq K_{a}\left|I_{z}\right|
$$

and

$$
|F(a)| \geq K_{a}\left|I_{z}\right|
$$

and $\lim _{a \rightarrow \infty} K_{a}=1$.

First we show how Theorem 1 follows from those two distribution inequalities. If $w \in I_{z}$ is in $E(a) \cap F(a)$, then

$$
A_{2 \delta(z)}(P(f \phi))(\lambda) \leq a^{1 / 2}\left\|f \circ \phi_{z}\right\|_{X} \inf _{w \in I_{z}} N_{X^{\prime}} \phi(w)
$$

and

$$
A_{2 \delta(z)}(P(g \psi)(\lambda)) \leq a^{1 / 2}\left\|g \circ \phi_{z}\right\|_{Y} \inf _{w \in I_{z}} N_{Y^{\prime}} \psi(w)
$$

Thus

$$
\begin{aligned}
A_{2 \delta(z)}( & P(f \phi))(\lambda) A_{2 \delta(z)}(P(g \psi)(\lambda) \\
& <a\left\|f \circ \phi_{z}\right\|_{X}\left\|g \circ \phi_{z}\right\|_{Y} \inf _{w \in I_{z}} N_{X^{\prime}}(\phi)(w) \inf _{w \in I_{z}} N_{Y^{\prime}}(\psi)(w) .
\end{aligned}
$$


So $E(a) \cap F(a)$ is a subset of

$$
\begin{aligned}
\left\{\lambda \in I_{z}:\right. & A_{2 \delta(z)}(P(f \phi))(\lambda) A_{2 \delta(z)}(P(g \psi))(\lambda) \\
& \left.<a\left\|f \circ \phi_{z}\right\|_{X}\left\|g \circ \phi_{z}\right\|_{Y} \inf _{w \in I_{z}} N_{X^{\prime}}(\phi)(w) \inf _{w \in I_{z}} N_{Y^{\prime}}(\psi)(w)\right\} .
\end{aligned}
$$

On the other hand, we have

$$
|E(a) \cap F(a)| \geq|E(a)|+|F(a)|-\left|I_{z}\right| \text {. }
$$

Since $\lim _{a \rightarrow \infty} K_{a}=1$, we have

$$
\begin{aligned}
& \mid\left\{\lambda \in I_{z}:\right. A_{2 \delta(z)}(P(f \phi))(\lambda) A_{2 \delta(z)}(P(g \psi)(\lambda) \\
&\left.<a\left\|f \circ \phi_{z}\right\|_{X}\left\|g \circ \phi_{z}\right\|_{Y} \inf _{w \in I_{z}} N_{X^{\prime}}(\phi)(w) \inf _{w \in I_{z}} N_{Y^{\prime}}(\psi)(w)\right\} \mid \\
& \quad \geq|E(a) \cap F(a)| \geq|E(a)|+|F(a)|-\left|I_{z}\right| \geq\left(2 K_{a}-1\right)\left|I_{z}\right|
\end{aligned}
$$

if $C_{a}=2 K_{a}-1$. This completes the proof of Theorem 1 .

Now we turn to the proof of our claim. For simplicity we will present only the details of the proof of (23). Using the same method we can prove $(24)$. The proof consists of three steps. Let $\chi_{E}$ denote the characteristic function of the subset $E$ of $\partial D$. In order to prove (23) we write $P(f \phi)$ as $P(f \phi)=P\left(\phi_{1}\right)+\left(P \phi_{2}\right)$ where $\phi_{1}=f\left(\chi_{2 I_{z}} \phi\right)$ and $\phi_{2}=f\left(\chi_{\partial D \backslash 2 I_{z}} \phi\right)$.

Step 1. There is a constant $C>0$ such that for all $t>0$,

$$
\begin{aligned}
\mid\left\{\lambda \in I_{z}:\right. & \left.A_{2 \delta(z)}\left(P\left(\phi_{1}\right)\right)<t\right\} \mid \\
& \geq\left(1-\frac{C\left\|f \circ \phi_{z}\right\|_{X} \inf _{w \in I_{z}} N_{X^{\prime}} \phi(w)}{t}\right)\left|I_{z}\right| .
\end{aligned}
$$

From the definition of the truncated Lusin area integral, we easily see that

$$
A_{2 \delta(z)}\left(P\left(\phi_{1}\right)\right) \leq A_{2 \delta(z)}\left(\phi_{1}\right)
$$

Thus

$$
\left\{\lambda \in I_{z}: A_{2 \delta(z)}\left(\phi_{1}\right)<t\right\} \subset\left\{\lambda \in I_{z}: A_{2 \delta(z)}\left(P\left(\phi_{1}\right)\right)<t\right\} .
$$

To prove (25) we need only to show

$$
\begin{aligned}
\mid\left\{\lambda \in I_{z}:\right. & \left.A_{2 \delta(z)}\left(\phi_{1}\right)<t\right\} \mid \\
& \geq\left(1-\frac{C\left\|f \circ \phi_{z}\right\|_{X} \inf _{w \in I_{z}} N_{X^{\prime}} \phi(w)}{t}\right)\left|I_{z}\right| .
\end{aligned}
$$


By the theorem of Marcinkiewicz and Zygmund, and the fact that the nontangential maximal function $M$ is of weak type $(1,1)$ then the truncated Lusin area integral $A_{\varepsilon} f(w)$ is also of weak type $(1,1)$. So we have, for $t>0$

$$
\left|\left\{\lambda \in I_{z}: A_{2 \delta(z)}\left(\phi_{1}\right) \geq t\right\}\right| \leq \frac{C}{t} \int_{\partial D}\left|\phi_{1}\right| d w=\frac{C}{t} \int_{2 I_{z}}|f(w) \phi(w)| d w .
$$

Since an elementary estimate shows that for $w \in 2 I_{z}, P(z, w)>C /\left|2 I_{z}\right|$, it follows that

$$
\begin{aligned}
\int_{2 I_{z}}|f(w) \phi(w)| d w & \leq C\left|I_{z}\right| \int|f(w) \phi(w)| P(z, w) d w \\
& =C\left|I_{z}\right| \int\left|f \circ \phi_{z}(w) \phi \circ \phi_{z}(w)\right| d w
\end{aligned}
$$

By the generalized Hölder inequality we have

$$
\int_{2 I_{z}}|f(w) \phi(w)| d w \leq C\left|I_{z}\right|\left\|f \circ \phi_{z}\right\|_{X}\left\|\phi \circ \phi_{z}\right\|_{X^{\prime}}
$$

Because for each $u \in I_{z}, z$ is in $\Gamma(u)$, we have

$$
\left|\left\{\lambda \in I_{z}: A_{2 \delta(z)}\left(\phi_{1}\right) \geq t\right\}\right| \leq \frac{C\left|I_{z}\right|}{t}\left\|f \circ \phi_{z}\right\|_{X} \inf _{w \in I_{z}} N_{X^{\prime}} \phi(w) .
$$

Thus

$\left|\left\{\lambda \in I_{z}: A_{2 \delta(z)}\left(\phi_{1}\right)<t\right\}\right| \geq\left(1-\frac{C\left\|f \circ \phi_{z}\right\|_{X} \inf _{w \in I_{z}} N_{X^{\prime}} \phi(w)}{t}\right)\left|I_{z}\right|$.

Step 2. On $I_{z}$,

$$
A_{2 \delta(z)}\left(P\left(\left(\phi_{2}\right)\right)(w) \leq C\left\|f \circ \phi_{z}\right\|_{X} \inf _{w \in I_{z}} N_{X^{\prime}}(\phi)(w)\right.
$$

for some $C>0$.

For $\phi_{2}$, we shall use a pointwise estimate of the norm of gradient of $P\left(\phi_{2}\right)$. Since $P\left(\phi_{2}\right)$ is analytic in $D$, we have

$$
\nabla\left(P\left(\phi_{2}\right)(w)\right)=\frac{1}{2 \pi} \int \frac{\bar{\xi} \phi_{2}(\xi)}{(1-w \bar{\xi})^{2}} d \xi
$$


Thus

$$
\left|\nabla P\left(\phi_{2}\right)(w)\right| \leq C \int \frac{\left|\phi_{2}(\xi)\right|}{|1-w \bar{\xi}|^{2}} d \xi \leq C \int_{\partial D \backslash 2 I_{z}} \frac{|f(\xi) \phi(\xi)|}{|1-w \bar{\xi}|^{2}} d \xi .
$$

On the other hand, there is a constant $C>0$ so that

$$
\left|\frac{1-(\xi, z)}{1-(\xi, w)}\right| \geq C
$$

for all $\xi$ in $\partial D \backslash 2 I_{z}$ and $w$ in $\Gamma_{u, 2 \delta(z)}$. Thus we obtain

$$
\left|\nabla\left(P \phi_{2}\right)(w)\right| \leq C \int_{\partial D \backslash 2 I_{z}} \frac{|f(\xi) \phi(\xi)|}{|1-\bar{z} \xi|^{2}} d \sigma(\xi) .
$$

Applying the generalized Hölder inequality yields

$$
\left|\nabla\left(P \phi_{2}\right)(u)\right| \leq \frac{C}{1-|z|^{2}}\left\|f \circ \phi_{z}\right\|_{X}\left\|\phi \circ \phi_{z}\right\|_{X^{\prime}} .
$$

Because $z$ belongs to $\Gamma(u)$, for any $u \in I_{z}$, the last factor on the right is no larger than $\mathrm{C} N_{X^{\prime}} \phi(u)$, and the desired inequality is established.

Step 3. This step will complete the proof of the distribution function inequality (23) by combining last two steps. Since $P(f \phi)=P\left(\phi_{1}\right)+$ $P\left(\phi_{2}\right)$, we have

$$
A_{2 \delta(z)}(P(f \phi))(w) \leq A_{2 \delta(z)}\left(\left(P \phi_{1}\right)\right)(w)+A_{2 \delta(z)}\left(P\left(\phi_{2}\right)\right)(w) .
$$

So for any $\lambda>0$,

$$
\bigcap_{i=1}^{2}\left\{w \in I_{z}: A_{2 \delta(z)}\left(\left(P \phi_{i}\right)\right) \leq \frac{\lambda}{2}\right\} \subset\left\{w \in I_{z}: A_{2 \delta(z)}(P(f \phi)) \leq \lambda\right\} .
$$

Let $E_{i}(a)$ be the subset of $I_{z}$ such that

$$
A_{2 \delta(z)}\left(\left(P \phi_{i}\right)\right) \leq a^{1 / 2}\left\|f \circ \phi_{z}\right\|_{X} \inf _{w \in I_{z}} N_{X^{\prime}} \phi(w)
$$

for $i \leq 2$.

Then we have

$$
\bigcap_{i=1}^{2} E_{i}\left(\frac{a}{2}\right) \subset E(a)
$$


If in Step 1 we choose that $t=a^{1 / 2}\left\|f \circ \phi_{z}\right\|_{X} \inf _{w \in I_{z}} N_{X^{\prime}} \phi(w)$, then we have

$$
\left|E_{1}\left(\frac{a}{2}\right)\right| \geq\left(1-C a^{-1 / 2}\right)\left|I_{z}\right|
$$

for a sufficiently large $a$.

By Step 2, for $a>0$ sufficiently large we have

$$
A_{2 \delta(z)}\left(P\left(\phi_{2}\right)\right)(u)<a^{1 / 2}\left\|f \circ \phi_{z}\right\|_{X} \inf _{w \in I_{z}} N_{X^{\prime}} \phi(w)
$$

everywhere on $I_{z}$, which implies $\left|E_{2}(a / 2)\right|=\left|I_{z}\right|$. So

$$
|E(a)| \geq\left(1-a^{-1 / 2} C\right)\left|I_{z}\right|
$$

This completes the proof of (23) if we choose $K_{a}=1-a^{-1 / 2} C$.

\section{Hilbert transform.}

In this section we apply the distribution function inequality (21) in Theorem 1 to get a sufficient condition for the boundedness of the Hilbert transform on two weighted $L^{p}$. Let $\|T\|_{p}^{z}$ denote the norm of the Hilbert transform $T$ from $L^{p}\left(u \circ \phi_{z}\right)$ to $L^{p}\left(v \circ \phi_{z}\right)$.

Given a Banach function space $X$, we will use $X^{\prime}$ to denote its associate space which is another Banach space.

Theorem 2. Let $u$ and $v$ two positive functions on the unit circle, and $1<p<\infty$. Suppose that $X$ and $Y$ are two Banach function spaces such that $N_{X^{\prime}}$ maps $L^{p^{\prime}}$ to $L^{p^{\prime}}$ and $N_{Y^{\prime}}$ maps $L^{p}$ to $L^{p}$. Then there is a constant $C>0$ such that

$$
\begin{aligned}
\left(\|T\|_{p}^{\lambda}\right)^{p} \leq C & \left(\sup _{z \in D}\left\|v^{1 / p} \circ \phi_{z}\right\|_{X}\left\|u^{-1 / p} \circ \phi_{z}\right\|_{Y}\right. \\
& \left.+\left(\sup _{z \in D} v(z)\left(u^{-1 /(p-1)}(z)\right)^{p-1}\right)^{1 / p}\right),
\end{aligned}
$$

for all $\lambda \in D$.

Proof. Since $T=-i P+i(I-P)$, it suffices to show that there is a constant $C>0$ such that

$$
\begin{aligned}
\left(\|P\|_{p}^{\lambda}\right)^{p} \leq C & \left(\sup _{z \in D}\left\|v^{1 / p} \circ \phi_{z}\right\|_{X}\left\|u^{-1 / p} \circ \phi_{z}\right\|_{Y}\right. \\
& \left.+\left(\sup _{z \in D} v(z)\left(u^{-1 /(p-1)}(z)\right)^{p-1}\right)^{1 / p}\right),
\end{aligned}
$$


for all $\lambda$. As $\left(v \circ \phi_{\lambda}\right)(z)=v\left(\phi_{\lambda}(z)\right)$ we see that $\sup _{z \in D}\left\|v^{1 / p} \circ \phi_{z}\right\|_{X}\left\|u^{-1 / p} \circ \phi_{z}\right\|_{Y}=\sup _{z \in D}\left\|v^{1 / p} \circ \phi_{\lambda} \circ \phi_{z}\right\|_{X}\left\|u^{-1 / p} \circ \phi_{\lambda} \circ \phi_{z}\right\|_{Y}$.

So it suffices to show

$$
\begin{aligned}
\left(\|P\|_{p}^{0}\right)^{p} \leq C & \left(\sup _{z \in D}\left\|v^{1 / p} \circ \phi_{z}\right\|_{X}\left\|u^{-1 / p} \circ \phi_{z}\right\|_{Y}\right. \\
& \left.+\left(\sup _{z \in D} v(z)\left(u^{-1 /(p-1)}(z)\right)^{p-1}\right)^{1 / p}\right) .
\end{aligned}
$$

This is equivalent to show

$$
\begin{aligned}
& \int|P(\phi)(w)|^{p} v(w) d w \\
& \qquad \begin{array}{l}
(30) \leq\left(\sup _{z \in D}\left\|v^{1 / p} \circ \phi_{z}\right\|_{X}\left\|u^{-1 / p} \circ \phi_{z}\right\|_{Y}\right. \\
\left.\quad+\left(\sup _{z \in D} v(z)\left(u^{-1 /(p-1)}(z)\right)^{p-1}\right)^{1 / p}\right) \int|\phi(w)|^{p} u(w) d w
\end{array}
\end{aligned}
$$

for all $\phi \in L^{p}(u)$. Let $\psi=\phi u^{1 / p}$. Then the above inequality is equivalent to the following inequality

$$
\begin{aligned}
& \int\left|P\left(\psi u^{-1 / p}\right)(w)\right|^{p} v(w) d w \\
& \leq C \sup _{z \in D}\left\|v^{1 / p} \circ \phi_{z}\right\|_{X}\left\|u^{-1 / p} \circ \phi_{z}\right\|_{Y} \\
& \left.\quad+\left(\sup _{z \in D} v(z)\left(u^{-1 /(p-1)}(z)\right)^{p-1}\right)^{1 / p}\right) \int|\psi(w)|^{p} d w
\end{aligned}
$$

for all $\psi \in L^{p}$. On the other hand,

$$
\int\left|P\left(\psi u^{-1 / p}\right)(w)\right|^{p} v(w) d w=\int\left|v^{1 / p}(w) P\left(\psi u^{-1 / p}\right)(w)\right|^{p} d w
$$

and the dual space of $L^{p}$ is $L^{p^{\prime}}$. Then

$$
\begin{array}{rl}
\int\left|P\left(\psi u^{-1 / p}\right)(w)\right|^{p} & v(w) d w \\
& =\sup _{\substack{\|h\|_{p^{\prime}} \leq 1 \\
h \in L^{p^{\prime}}}}\left|\int v^{1 / p}(w) P\left(\psi u^{-1 / p}\right)(w) \overline{h(w)} d w\right| .
\end{array}
$$


So we need only to estimate the pairing

$$
\int v^{1 / p}(w) P\left(\psi u^{-1 / p}\right)(w) \overline{h(w)} d w
$$

Now

$$
\begin{aligned}
\int v^{1 / p}(w) P\left(\psi u^{-1 / p}\right)(w) \overline{h(w)} & \\
& =\int P\left(\psi u^{-1 / p}\right)(w) \overline{P\left(v^{1 / p} h\right)(w)} d w .
\end{aligned}
$$

Using the Littlewood-Paley formula [10], we have

$$
\begin{aligned}
\int P(\psi & \left.u^{-1 / p}\right)(w) \overline{P\left(v^{1 / p} h\right)(w)} d w \\
= & P\left(\psi u^{-1 / p}\right)(0) \overline{P\left(v^{1 / p} h\right)(0)} \\
& +\iint\left\langle\nabla \left( P\left(u^{-1 / p} \psi\right)(z), \nabla\left(P\left(v^{1 / p} h\right)(z)\right\rangle \log \frac{1}{|z|} d A(z) .\right.\right.
\end{aligned}
$$

Define

$$
\operatorname{Term}_{I}=\iint_{|z| \geq 1 / 2}\left\langle\nabla \left( P\left(u^{-1 / p} \psi\right)(z), \nabla\left(P\left(v^{1 / p} h\right)(z)\right\rangle \log \frac{1}{|z|} d A(z) .\right.\right.
$$

and

$$
\operatorname{Term}_{I I}=\iint_{|z|<1 / 2}\left\langle\nabla \left( P\left(u^{-1 / p} \psi\right)(z), \nabla\left(P\left(v^{1 / p} h\right)(z)\right\rangle \log \frac{1}{|z|} d A(z) .\right.\right.
$$

It is easy to verify that

$$
\left|P\left(\psi u^{-1 / p}\right)(0) \overline{P\left(v^{1 / p} h\right)(0)}\right| \leq C\left(v(0) u^{-1 /(p-1)}(0)^{p-1}\right)^{1 / p}\|\psi\|_{p}\|h\|_{p^{\prime}},
$$

and

$$
\begin{aligned}
& \mid\left\langle\nabla \left( P\left(u^{-1 / p} \psi\right)(z), \nabla\left(P\left(v^{1 / p} h\right)(z)\right\rangle \mid\right.\right. \\
& \quad \leq C\left(v(0) u^{-1 /(p-1)}(0)^{p-1}\right)^{1 / p}\|\psi\|_{p}\|h\|_{p^{\prime}}
\end{aligned}
$$

for $|z| \leq 1 / 2$. So we need only an estimate of $\operatorname{Term}_{I}$. We claim that there is a constant $C>0$ such that

$$
\left|\operatorname{Term}_{I}\right| \leq C \sup _{z \in D}\left\|v^{1 / p} \circ \phi_{z}\right\|_{X}\left\|u^{-1 / p} \circ \phi_{z}\right\|_{Y}\|\psi\|_{p}\|h\|_{p^{\prime}} .
$$


So by (31), $P$ is bounded from $L^{p}(u)$ to $L^{p}(v)$ and its norm is bounded by

$$
C\left(\sup _{z \in D}\left\|v^{1 / p} \circ \phi_{z}\right\|_{X}\left\|u^{-1 / p} \circ \phi_{z}\right\|_{Y}+\left(\sup _{z \in D} v(z)\left(u^{-1 /(p-1)}(z)\right)^{p-1}\right)^{1 / p}\right) .
$$

Now we turn to the proof of the claim. Fix an $a>0$ for which the first distribution function inequality in Theorem 1 holds. For $w \in \partial D$, let $\rho(w)$ denote the maximum of those numbers $\varepsilon$ for which

$$
\begin{aligned}
& A_{\varepsilon}\left(P\left(u^{-1 / p} \psi\right)\right)(w) A_{\varepsilon}\left(P\left(v^{1 / p} h\right)\right)(w) \\
& \quad \leq a \sup _{z \in D}\left\|v^{1 / p} \circ \phi_{z}\right\|_{X}\left\|u^{-1 / p} \circ \phi_{z}\right\|_{Y} N_{X^{\prime}} h(w) N_{Y^{\prime}} \psi(w) .
\end{aligned}
$$

Thus

$$
\begin{aligned}
& \int_{\partial D} A_{\rho(w)}\left(P\left(u^{-1 / p} \psi\right)\right)(w) A_{\rho(w)}\left(P\left(v^{1 / p} h\right)\right)(w) d w \\
& \quad \leq a \sup _{z \in D}\left\|v^{1 / p} \circ \phi_{z}\right\|_{X}\left\|u^{-1 / p} \circ \phi_{z}\right\|_{Y} \int N_{X^{\prime}} h(w) N_{Y^{\prime}} \psi(w) .
\end{aligned}
$$

By the Hölder inequality, we have

$$
\begin{aligned}
\int_{\partial D} A_{\rho(w)} & \left(P\left(u^{-1 / p} \psi\right)\right)(w) A_{\rho(w)}\left(P\left(v^{1 / p} h\right)\right)(w) d w \\
\leq & a \sup _{z \in D}\left\|v^{1 / p} \circ \phi_{z}\right\|_{X}\left\|u^{-1 / p} \circ \phi_{z}\right\|_{Y}\left\|N_{X^{\prime}} h\right\|_{p^{\prime}}\left\|N_{Y^{\prime}} g\right\|_{p} .
\end{aligned}
$$

Since $N_{X^{\prime}}$ maps $L^{p^{\prime}}$ to $L^{p^{\prime}}$ and $N_{Y^{\prime}}$ maps $L^{p}$ to $L^{p}$, we have

$$
\begin{aligned}
\int_{\partial D} A_{\rho(w)}\left(P\left(u^{-1 / p} \psi\right)\right)(w) A_{\rho(w)}\left(P\left(v^{1 / p} h\right)\right)(w) d w \\
\leq C \sup _{z \in D}\left\|v^{1 / p} \circ \phi_{z}\right\|_{X}\left\|u^{-1 / p} \circ \phi_{z}\right\|_{Y}\|\psi\|_{p}\|h\|_{p^{\prime}} .
\end{aligned}
$$

On the other hand, let $\chi_{w}(z)$ denote the characteristic function of $\Gamma_{w, \rho(w)}$, we have

$$
\begin{aligned}
& \int_{\partial D} A_{\rho(w)}\left(P\left(u^{-1 / p} \psi\right)\right)(w) A_{\rho(w)}\left(P\left(v^{1 / p} h\right)\right)(w) d w \\
& =\int_{\partial D}\left(\int_{\Gamma_{w, \rho(w)}} \mid \nabla\left(\left.P\left(u^{-1 / p} \psi\right)(z)\right|^{2} d A(z)\right)^{1 / 2}\right. \\
& \quad \cdot\left(\int_{\Gamma_{w, \rho(w)}} \mid \nabla\left(\left.P\left(v^{1 / p} h\right)(z)\right|^{2} d A(z)\right)^{1 / 2} d \omega\right. \\
& \geq \int_{|z|>1 / 2} \int_{\partial D} \chi_{w}(z)\left|\nabla\left(P\left(u^{-1 / p} \psi\right)\right)(z)\right|\left|\nabla\left(P\left(v^{1 / p} h\right)\right)(z)\right| d w d A(z) .
\end{aligned}
$$


Now the distribution function inequality (21) tells us that $\rho(w) \geq 2(1-$ $|z|)$ on a subset of $I_{z}$ whose measure is at least $C_{a}(1-|z|)$. If $\rho(w) \geq$ $2(1-|z|)$, then $z$ is in $\Gamma_{w, \rho(w)}$. Thus $\chi_{w}(z)=1$ on a subset of $I_{z}$ of measure at least $C_{a}(1-|z|)$. Combining this observation with previous inequality, we obtain

$$
\begin{aligned}
& \int_{\partial D} A_{\rho(w)}\left(P\left(u^{-1 / p} \psi\right)\right)(w) A_{\rho(w)}\left(P\left(v^{1 / p} h\right)\right)(w) d w \\
& \quad \geq C_{a} \int_{|z|>1 / 2} \mid \nabla\left(P ( u ^ { - 1 / p } \psi ) ( z ) | | \nabla \left(P\left(v^{1 / p} h\right)(z) \mid(1-|z|) d A(z)\right.\right. \\
& \quad \geq C_{a}\left|\operatorname{Term}_{I}\right|
\end{aligned}
$$

So

$$
\left|\operatorname{Term}_{I}\right| \leq C \sup _{z \in D}\left\|v^{1 / p} \circ \phi_{z}\right\|_{X}\left\|u^{-1 / p} \circ \phi_{z}\right\|_{Y}\|\psi\|_{p}\|h\|_{p^{\prime}} .
$$

This completes the proof of the theorem.

Corollary 1. Let $X$ and $Y$ be either Orlicz spaces or Lorentz spaces. Suppose that $M_{X^{\prime}}$ maps $L^{p^{\prime}}$ to $L^{p^{\prime}}$ and $M_{Y^{\prime}}$ maps $L^{p}$ to $L^{p}$. Then the Hilbert transform $T$ is uniformly bounded from $L^{p}\left(v \circ \phi_{z}\right)$ to $L^{p}\left(v \circ \phi_{z}\right)$ for all $z \in D$.

Proof. Since $X$ and $Y$ are either Orlicz spaces or Lorentz spaces, by Proposition 1, the maximal operators $M_{X^{\prime}}$ and $M_{Y^{\prime}}$ dominate the nontangential maximal operators $N_{X^{\prime}}$ and $N_{Y^{\prime}}$ respectively. So $N_{X^{\prime}}$ maps $L^{p^{\prime}}$ to $L^{p^{\prime}}$ and $N_{Y^{\prime}}$ maps $L^{p}$ to $L^{p}$. Also it was shown in [19] that if $M_{X^{\prime}}$ maps $L^{p^{\prime}}$ to $L^{p^{\prime}}$, then

$$
\|f\|_{p} \leq C\|f\|_{X},
$$

for any $f \in X$. Then

$$
\sup _{z \in D} v(z)\left(u^{-1 /(p-1)}(z)\right)^{p-1} \leq C\left(\sup _{z \in D}\left\|v^{1 / p} \circ \phi_{z}\right\|_{X}\left\|u^{-1 /(p-1)} \circ \phi_{z}\right\|_{Y}\right)^{p} .
$$

So the corollary follows immediately from Theorem 2 .

A particular example is when $X=L^{p r}$ and $Y=L^{p^{\prime} r}$, where $r>1$. In this case the associate spaces are $X^{\prime}=L^{(p r)^{\prime}}$ and $Y^{\prime}=L^{\left(p^{\prime} r\right)^{\prime}}$ whose corresponding maximal operators are given by

$$
M_{X^{\prime}} f(x)=\sup _{x \in I}\left(\frac{1}{|I|} \int_{I}|f(y)|^{(p r)^{\prime}} d y\right)^{1 /(p r)^{\prime}}
$$


and

$$
M_{Y^{\prime}} f(x)=\sup _{x \in I}\left(\frac{1}{|I|} \int_{I}|f(y)|^{\left(p^{\prime} r\right)^{\prime}} d y\right)^{1 /\left(p^{\prime} r\right)^{\prime}}
$$

which are bounded on $L^{p^{\prime}}$ and $L^{p}$, respectively. By Proposition 1 , the maximal operator $M_{X}$ dominates the nontangential maximal operator $N_{X}$ if $X$ is an Orlicz space. So we have the following corollary.

Corollary 2. Let $1<p<\infty$, and suppose that $(u, v)$ is a pair of weights such that for some $r>1$,

$$
\sup _{z \in D} v^{r}(z)\left(u^{-r /(p-1)}(z)\right)^{p-1}<\infty .
$$

Then the Hilbert transform is bounded from $L^{p}(u)$ to $L^{p}(v)$.

The condition in Corollary 2 is quite close to the necessary condition that the Hilbert transform is uniformly bounded from $L^{p}\left(u \circ \phi_{\lambda}\right)$ to $L^{p}\left(u \circ \phi_{\lambda}\right)$ for $\lambda \in D$.

Proposition 2. Let $u$ and $v$ be two positive functions on the unit circle. Let $1<p<\infty$. If the Hilbert transform is uniformly bounded from $L^{p}\left(u \circ \phi_{\lambda}\right)$ to $L^{p}\left(u \circ \phi_{\lambda}\right)$ for $\lambda \in D$, then

$$
\sup _{z \in D} v(z)\left(u^{-1 /(p-1)}(z)\right)^{p-1}<\infty
$$

Proof. For a fixed $\lambda \in D$, let $P_{0}$ be the operator

$$
P-z P \bar{z}
$$

from $L^{p}(u)$ to $L^{p}(v)$. It is easy to check that

$$
P_{0} f=\left(\int f(x) e_{0}(x) d x\right) e_{0},
$$

for $f \in L^{p}\left(u \circ \phi_{\lambda}\right)$ where $e_{0}(z)=1$. Since the Hilbert transform is unformly bounded from $L^{p}\left(u \circ \phi_{\lambda}\right)$ to $L^{p}\left(v \circ \phi_{\lambda}\right), P$ is also uniformly bounded from $L^{p}\left(u \circ \phi_{\lambda}\right)$ to $L^{p}\left(v \circ \phi_{\lambda}\right)$. So there is a constant $C>0$ such that

$$
\left\|P_{0}\right\|_{L^{p}\left(u \circ \phi_{\lambda}\right) \rightarrow L^{p}\left(v \circ \phi_{\lambda}\right)} \leq C
$$


for all $\lambda \in D$. On the other hand, the norm of $P_{0}$ from $L^{p}\left(u \circ \phi_{\lambda}\right)$ to $L^{p}\left(v \circ \phi_{\lambda}\right)$ is $\left\|u^{-1 / p} \circ \phi_{\lambda}\right\|_{p^{\prime}}\left\|v \circ \phi_{\lambda}\right\|_{1}^{1 / p}$. This completes the proof of the proposition.

We can improve Corollary 2 using the scale of Lorentz spaces: if $X=L^{p r, \infty}$, then $X^{\prime}=L^{(p r)^{\prime}, 1}$ and $M_{X^{\prime}}$ is bounded on $L^{p^{\prime}}$. By Proposition 1 , the maximal operator $M_{X}$ dominates the nontangential maximal operator $N_{X}$ if $X$ is a Lorentz space. Hence we have the following corollary.

Corollary 3. Let $1<p<\infty$, and $1<r<\infty$. Suppose that $(u, v)$ is a pair of weights such that

$$
\begin{aligned}
& \sup _{z \in D}\left(\sup _{t>0}\left(\int_{\{v(x)>t\}} t^{r} P(z, x) d x\right)\right) \\
& \cdot\left(\sup _{t>0}\left(\int_{\left\{u^{-1}(x)>t\right\}} t^{-r /(p-1)} P(z, x) d x\right)^{p-1}\right)<\infty .
\end{aligned}
$$

Then the Hilbert transform is bounded from $L^{p}(u)$ to $L^{p}(v)$.

More interesting examples are provided by the theory of Orlicz spaces. We have the following theorem which improves Corollary 2. In particular, if Young functions $\Phi$ and $\Psi$ are in the following forms

$$
\Phi(t)=t^{p} \log ^{p-1+\delta}(1+t),
$$

and

$$
\Psi(t)=t^{p^{\prime}} \log p^{p^{\prime}-1+\delta}(1+t)
$$

or weaker ones

$$
\Phi(t)=t^{p} \log ^{p-1}(1+t)(\log \log (1+t))^{p-1+\delta}
$$

and

$$
\Psi(t)=t^{p^{\prime}} \log p^{p^{\prime}-1}(1+t)(\log \log (1+t))^{p^{\prime}-1+\delta},
$$

then the correspoding Orlicz spaces satisfy conditions in the following theorem.

Theorem 3. Let $1<p<\infty$, and let $\Phi(t)$ and $\Psi(t)$ be Young functions satisfying $\Delta_{2}$-condition such that

$$
\int_{c}^{\infty}\left(\frac{t^{p}}{\Phi(t)}\right)^{p^{\prime}-1} \frac{d t}{t}<\infty \quad \text { and } \quad \int_{c}^{\infty}\left(\frac{t^{p^{\prime}}}{\Psi(t)}\right)^{p-1} \frac{d t}{t}<\infty
$$


for some positive constant $c$. Let $(u, v)$ be a pair of weights such that

$$
\sup _{z \in D}\left\|v^{1 / p} \circ \phi_{z}\right\|_{L^{\Phi}}\left\|u^{-1 / p} \circ \phi_{z}\right\|_{L^{\Psi}}<\infty .
$$

Then the Hilbert transform $T$ is bounded from $L^{p}(u)$ to $L^{p}(v)$.

Proof. Let $X=L^{\Phi}$ and $Y=L^{\Psi}$. To prove the theorem, by Theorem 2, we need to show that $N_{X^{\prime}}$ maps $L^{p^{\prime}}$ to $L^{p^{\prime}}$ and $N_{Y^{\prime}}$ maps $L^{p}$ to $L^{p}$. By Proposition 1, we see that $M_{X^{\prime}}$ and $M_{Y^{\prime}}$ dominate $N_{X^{\prime}}$ and $N_{Y^{\prime}}$, respectively. On the other hand, it is shown in [19] that $M_{X^{\prime}}$ maps $L^{p}$ to $L^{p}$ and $M_{Y^{\prime}}$ maps $L^{p^{\prime}}$ to $L^{p^{\prime}}$. This completes the proof of the theorem.

\section{Invariant $A_{\infty}$ weights.}

$A_{\infty}$ weights are introduced in connection with several problems in harmonic analysis by Muckenhoupt [15] and Coifman-Fefferman [3]. First we introduce the $A_{\infty}$ condition as [3] and [14]. A weight function $v$ on the unit circle is in $A_{\infty}$ if there are positive constants $C, \delta>0$ so that given any arc $I$ and any measurable subset $E \subset I$

$$
\frac{\int_{E} v(w) d w}{\int_{I} v(w) d w} \leq C\left(\frac{|E|}{|I|}\right)^{\delta} .
$$

There are many characterizations of $A_{\infty}$ weights [9]. A characterization of $A_{\infty}$ similar to the $A_{p}$ condition is found in [11]. That is, a weight function $v$ is in $A_{\infty}$ if and only if

$$
\sup _{I} \frac{1}{|I|} \int_{I} v(w) d w\left(\frac{1}{|I|} \exp \left(\int_{I} \log v^{-1}(w) d w\right)\right)<\infty .
$$

But the $A_{\infty}$ condition is not Möbius invariant in the sense

$$
\sup _{z \in D} \sup _{I} \frac{1}{|I|} \int_{I} v \circ \phi_{z}(w) d w\left(\frac{1}{|I|} \exp \left(\int_{I} \log v^{-1} \circ \phi_{z}(w) d w\right)\right)<\infty .
$$

We define a weight $v$ to be an invariant $A_{\infty}$ weight if

$$
\sup _{z \in D} v(z) \exp (-(\log v)(z))<\infty .
$$


The invariant $A_{\infty}$ weights were first studied by Wolff in [26]. He [26] showed that a weight $v$ is an invariant $A_{\infty}$ weight if and only if for any $\alpha>0$ there is a $\beta>0$ : for any subarc $I \subset \partial D$ and subset $E \subset I$, then

$$
\int_{E} v(w) P(z, w) d w \leq \beta \int_{I} v(w) P(z, w) d w
$$

implies that

$$
\int_{E} P(z, w) d w \leq \alpha \int_{I} P(z, w) d w,
$$

for all $z \in D$. So comparing an equivalent condition to $A_{\infty}$ ([3, Lemma 5]) we see that invariant $A_{\infty}$ weights have a certain invariant property.

If two weights are invariant $A_{\infty}$ weights, first we will show a necessary and sufficient conditions for the Hilbert transform to be uniformly bounded between two weighted spaces.

Theorem 4. Suppose that both $v$ and $u^{-1 /(p-1)}$ are invariant $A_{\infty}$ weights. The invariant $A_{p}$ condition

$$
\sup _{z \in D} v(z)\left(u^{-1 /(p-1)}(z)\right)^{p-1}<\infty
$$

is a necessary and sufficient condition for the Hilbert transform to be uniformly bounded from $L^{p}\left(u \circ \phi_{z}\right)$ to $L^{p}\left(v \circ \phi_{z}\right)$ for all $z \in D$.

Proof. By Proposition 2, we see that the condition in Theorem 4 is a necessary condition that the Hilbert transform is uniformly bounded from $L^{p}\left(u \circ \phi_{z}\right)$ to $L^{p}\left(v \circ \phi_{z}\right)$ for all $z \in D$.

We need only to show that the condition in Theorem 4 is sufficient. As pointed out in [26], the invariant $A_{\infty}$ weight condition is equivalent to that $v(w) P(z, w) d w$ is comparable to $P(z, w) d w$ in the sense of [3] uniformly over $z \in D$. So the equivalent conditions of [3, Lemma 5] are valid for the measures $v(w) P(z, w) d w$ and $P(z, w) d w$ uniformly over $z \in D$. So there are positive constants $B$ and $r>1$ such that

$$
v^{r}(z) \leq B(v(z))^{r}
$$

for all $z \in D$.

Since both $v$ and $u^{-1 /(p-1)}$ are invariant $A_{\infty}$ weights, we can choose a constant $r>1$ so that

$$
\begin{aligned}
v^{r}(z) & \leq B(v(z))^{r}, \\
u^{-r /(p-1)}(z) & \leq B\left(u^{-1 /(p-1)}(z)\right)^{r},
\end{aligned}
$$


for all $z \in D$. Thus

$$
v^{r}(z)\left(u^{-r /(p-1)}(z)\right)^{p-1} \leq B^{2}(v(z))^{r}\left(u^{-1 /(p-1)}(z)\right)^{r},
$$

for all $z \in D$. If $u$ and $v$ satisfy

$$
\sup _{z \in D} v(z)\left(u^{-1 /(p-1)}(z)\right)^{p-1}<\infty
$$

then they also satisfy

$$
\sup _{z \in D} v^{r}(z)\left(u^{-r /(p-1)}(z)\right)^{p-1}<\infty .
$$

By Corollary 2, the Hilbert transform $T$ is uniformly bounded from $L^{p}\left(u \circ \phi_{z}\right)$ to $L^{p}\left(v \circ \phi_{z}\right)$. This completes the proof of Theorem 4 .

What is the difference between $A_{\infty}$ weights and invariant $A_{\infty}$ weights? The following theorem answers the question completely.

Let $f(z)$ and $g(z)$ be two nonnegative functions. We use $f(z) \cong$ $g(z)$ to denote that there are two positive constants $C_{1}$ and $C_{2}$ such that

$$
C_{1} f(z) \leq g(z) \leq C_{2} f(z)
$$

for any $z$.

For each $z=r e^{i t} \in D$, recall $I_{z}$ as in Section 2

$$
I_{z}=\left\{e^{i \theta} \in \partial D:|\theta-t| \leq \frac{1-r}{2}\right\}
$$

Theorem 5. Let $v$ be a positive function on the unit circle. Then $v$ is an invariant $A_{\infty}$ weight if and only if $v$ is an $A_{\infty}$ weight, and

$$
v(z) \cong \frac{1}{\left|I_{z}\right|} \int_{I_{z}} v(w) d w,
$$

for all $z \in D$.

Proof. Suppose that $v$ is an invariant $A_{\infty}$ weight. First we will prove that $\log v$ is in BMO. By the Jensen inequality, we have

$$
\begin{aligned}
\exp \left(\int_{D}(\log v-(\log v)(z)) P_{z}\left(e^{i \theta}\right) d \theta\right) \\
\leq \int_{D} \exp (\log v-(\log v)(z)) P_{z}\left(e^{i \theta}\right) d \theta \leq C .
\end{aligned}
$$


Hence

$$
\begin{array}{r}
\exp \left(\int_{D}(\log v-(\log v)(z))^{+} P_{z}\left(e^{i \theta}\right) d \theta\right) \\
\leq \exp \left(\int_{D}(\log v-(\log v)(z)) P_{z}\left(e^{i \theta}\right) d \theta\right) \leq C,
\end{array}
$$

and

$$
\begin{array}{r}
\exp \left(\int_{D}(\log v-(\log v)(z))^{-} P_{z}\left(e^{i \theta}\right) d \theta\right) \\
\leq \exp \left(\int_{D}(\log v-(\log v)(z)) P_{z}\left(e^{i \theta}\right) d \theta\right) \leq C .
\end{array}
$$

because

$$
\int_{D}(\log v-(\log v)(z))^{+} P_{z}\left(e^{i \theta}\right) d \theta=\int_{D}(\log v-(\log v)(z))^{-} P_{z}\left(e^{i \theta}\right) d \theta .
$$

On the other hand,

$$
\begin{aligned}
\int_{D}|\log v-(\log v)(z)| P_{z}\left(e^{i \theta}\right) d \theta= & \int_{D}(\log v-(\log v)(z))^{+} P_{z}\left(e^{i \theta}\right) d \theta \\
& +\int_{D}(\log v-(\log v)(z))^{-} P_{z}\left(e^{i \theta}\right) d \theta \\
\leq & 2 \log C .
\end{aligned}
$$

So $\log v$ is in BMO. For each interval $I$, there is a point $z_{I}$ such that $I=I_{z_{I}}$. We use $v_{I}$ to denote the average of $v$ over $I$. Since $\log v$ is in $\mathrm{BMO}$, we have

$$
\begin{aligned}
\left|(\log v)_{I}-(\log v)(z)\right| & \leq\left|\frac{1}{\left|I_{z}\right|} \int_{I_{z}}(\log v-(\log v)(z)) d \theta\right| \\
& \leq \frac{1}{\left|I_{z}\right|} \int_{I_{z}}|\log v-(\log v)(z)| d \theta \\
& \leq C \int_{I_{z}}|\log v-(\log v)(z)| P_{z}\left(e^{i \theta}\right) d \theta \\
& \leq C\|\log v\|_{\mathrm{BMO}} .
\end{aligned}
$$

Hence

$$
v_{I} \leq v\left(z_{I}\right) \leq C e^{(\log v)(z)-(\log v)_{I}+(\log v)_{I}} \leq C e^{(\log v)_{I}}
$$


So $v$ is an $A_{\infty}$ weight. In addition,

$$
v(z) \leq C e^{(\log v)(z)-(\log v)_{I}+(\log v)_{I}} \leq C e^{(\log v)_{I}} .
$$

By the Jensen inequality we have

$$
v(z) \leq C v_{I}
$$

Conversely suppose that $v$ is an $A_{\infty}$ weight and satisfy

$$
v(z) \cong \frac{1}{\left|I_{z}\right|} \int_{I_{z}} v(w) d w
$$

for all $z \in D$.

Since $v$ is an $A_{\infty}$ weight, $\log v$ is in BMO and

$$
\frac{1}{\left|I_{z}\right|} \int_{I_{z}} v\left(e^{i \theta}\right) d \theta \leq C \exp \left(\frac{1}{\left|I_{z}\right|} \int_{I_{z}} \log v\left(e^{i \theta}\right) d \theta\right)
$$

for some $C>0$. On the other hand,

$$
\begin{aligned}
\frac{1}{\left|I_{z}\right|} \int_{I_{z}}(\log v-(\log v)(z)) d \theta & \leq \frac{1}{\left|I_{z}\right|} \int_{I_{z}}|\log v-(\log v)(z)| d \theta \\
& \leq C \int_{I_{z}}|\log v-(\log v)(z)| P_{z}\left(e^{i \theta}\right) d \theta \\
& \leq C\|\log v\|_{\mathrm{BMO}} .
\end{aligned}
$$

Hence

$$
\frac{1}{\left|I_{z}\right|} \int_{I_{z}}(\log v) d \theta \leq C\|\log v\|_{\mathrm{BMO}}+(\log v)(z)
$$

Thus

$$
\begin{aligned}
v(z) & \leq C \frac{1}{\left|I_{z}\right|} \int_{I_{z}} v\left(e^{i \theta}\right) d \theta \\
& \leq C \exp \left(\frac{1}{\left|I_{z}\right|} \int_{I_{z}}(\log v)\left(e^{i \theta}\right) d \theta\right) \\
& \leq C e^{C_{1}\|\log v\|_{\mathrm{BMO}}+(\log v)(z)} \\
& \leq C e^{(\log v)(z)} .
\end{aligned}
$$


So $v(z)$ is an invariant $A_{\infty}$ weight. This completes the proof of Theorem 5 .

We remark that $A_{\infty}$ condition is not comparable with

$$
v(z) \cong \frac{1}{\left|I_{z}\right|} \int_{I_{z}} v(w) d w
$$

for all $z \in D$. There is an $A_{\infty}$ weight $v$ which $v(z)$ is not equivalent to $\int_{I_{z}} v(w) d w /\left|I_{z}\right|$. As in [26], for example $v\left(e^{i \theta}\right)=|\theta|$ is in $A_{\infty}$. But it is not in invariant $A_{\infty}$. So by Theorem $5, v(z)$ is not equivalent to $\int_{I_{z}} v(w) d w /\left|I_{z}\right|$.

Also there is a function $v>0$ such that $v(z)$ is equivalent to $\int_{I_{z}} v(w) d w /\left|I_{z}\right|$. But $v$ is even not in $A_{\infty}$, see examples in [24].

If $v$ is in $A_{2}$, then $v(z)$ is equivalent to $\int_{I_{z}} v(w) d w /\left|I_{z}\right|$. Thus an $A_{2}$ weight is also an invariant $A_{\infty}$ weight. So a weight function $v$ is an $A_{2}$ weight if and only if both $v$ and $v^{-1}$ are invariant $A_{\infty}$ weights. Therefore invariant $A_{\infty}$ weights are somehow $1 / 2-A_{2}$ weights. But there are invariant $A_{\infty}$ weights which are not in $A_{2}$ [26].

Recently Fefferman-Kenig-Pipher [7] characterized $A_{\infty}$ weights in terms of Carleson measures, which is very close to $\log v \in$ BMO. To state their result more precisely we consider weights $v$ on the real line $\mathbb{R}$. Let $\Phi_{t}(x)=c t^{-1 / 2} e^{-|x|^{2} / t}$, fix a function $v$ which verifies the doubling condition

$$
\int_{\left|x-x_{0}\right|<2 t} v(\theta) d \theta \leq \rho \int_{\left|x-x_{0}\right|<t} v(\theta) d \theta,
$$

for some $\rho>1$. The heat extension of $v$ will be defined by $v(x, t)=$ $\left(v \star \Phi_{t}\right)(x)$, and $\nabla v(x, t)$ will denote the spatial gradient. FeffermanKenig-Pipher showed that $v \in A_{\infty}(R)$ if and only if for all $x_{0} \in \mathbb{R}$ and $t>0$,

$$
\frac{1}{t} \int_{0}^{t^{2}} \int_{\left|x-x_{0}\right|<t} \frac{|\nabla v(x, s)|^{2}}{(v(x, s))^{2}} d x d s<C .
$$

They [7] made a remark that the above result with the harmonic (Poisson) extension in place of the heat extension would not characterize $A_{\infty}(\mathbb{R})$, for the Poisson kernel need not have sufficiently rapid decay.

However, using harmonic extension of weights we will characterize invariant $A_{\infty}$ weights in terms of Carleson measures.

Theorem 6. Let $v$ be a positive function on the unit circle. Then $v$ is an invariant $A_{\infty}$ weight if and only if $\left(|\nabla v(z)|^{2} / v(z)^{2}\right)\left(1-|z|^{2}\right)$ is a 
Carleson measure, i.e.,

$$
\int_{S(I)}\left(\frac{|\nabla v(z)|^{2}}{v(z)^{2}}\right)\left(1-|z|^{2}\right) d A(z) \leq C|I|
$$

for any subarcs I of the unit circle, where

$$
S(I)=\left\{r e^{i t} \in D: 1-r<|I|, e^{i t} \in I\right\} .
$$

Proof. Since $v(z)$ is harmonic on $D$, we have

$$
-\Delta \log (v(z))=\frac{|\nabla v(z)|^{2}}{(v(z))^{2}} .
$$

By Green's formula, we obtain

$$
\log (v(z))-(\log v)(z)=-\int_{D} \log \left|\frac{1-\bar{z} w}{z-w}\right| \Delta(\log (v(w))) d A(w) .
$$

So

$$
\log (v(z))-(\log v)(z)=\int_{D} \log \left|\frac{1-\bar{z} w}{z-w}\right| \frac{|\nabla v(w)|^{2}}{(v(w))^{2}} d A(w) .
$$

For $1 / 2<|w|<1$, it is easy to check that $\log |w|^{-1} \cong\left(1-|w|^{2}\right)$. Also since $v(w)$ is harmonic on $D,|\nabla v(w)|^{2} /(v(w))^{2}$ is bounded by a constant $M$ for all $w$ with $|w| \leq 1 / 2$. By the above equation, we have

$\log (v(z))-(\log v)(z) \leq \int_{|w|>1 / 2}\left(1-\left|\frac{1-\bar{z} w}{z-w}\right|^{-2}\right) \frac{|\nabla v(w)|^{2}}{(v(w))^{2}} d A(w)+M$

and

$$
\log (v(z))-(\log v)(z) \geq \int_{|w|>1 / 2}\left(1-\left|\frac{1-\bar{z} w}{z-w}\right|^{-2}\right) \frac{|\nabla v(w)|^{2}}{(v(w))^{2}} d A(w) .
$$

By [10], $\left(1-|w|^{2}\right)|\nabla v(w)|^{2} d A(w) /(v(w))^{2}$ is a Carleson measure if and only if

$$
\sup _{z \in D} \int_{D} \frac{\left(1-|z|^{2}\right)}{|1-\bar{z} w|^{2}} \frac{|\nabla v(w)|^{2}}{|v(w)|^{2}}\left(1-|w|^{2}\right) d A(w)<\infty
$$


This is equivalent to

$$
\log (v(z))-(\log v)(z) \leq C,
$$

for all $z \in D$. This completes the proof of Theorem 6 .

Another important property of $A_{\infty}$ is that

$$
A_{\infty}=\bigcup_{p>0} A_{p}
$$

We will show that invariant $A_{\infty}$ weights have such property also.

Theorem 7. Any weight function $v$ satisfying invariant $A_{\infty}$ already satisfies invariant $A_{p}$ for some $p<\infty$.

Proof. Since $v$ is an invariant $A_{\infty}$ weight, we have

$$
\log (v(z))-(\log v)(z) \leq C .
$$

By the Jensen inequality, we also have

$$
\log (v(z))-(\log v)(z) \geq 0 .
$$

On the other hand, by Theorem 5 , we see that $v$ is an $A_{\infty}$ weight. It follows from [10] that $\log v$ is in BMO. By the theorem of John and Nirenberg [10], there exist positive constants $C_{1}$ and $C_{2}$ such that

$$
|\{w \in \partial D:|\log v(w)-(\log (v))(0)|>\lambda\}| \leq C_{1} e^{-C_{2} \lambda /\|\log v\|_{\text {вмо }}},
$$

for $\lambda>0$. Let $\phi_{z}(w)$ denote the Möbius map on the unit disk. Then $\left\|\log v \circ \phi_{z}\right\|_{\mathrm{BMO}}=\|\log v\|_{\mathrm{BMO}}$. Therefore

$\left|\left\{w \in \partial D:\left|\log v \circ \phi_{z}(w)-\log \left(v \circ \phi_{z}\right)(0)\right|>\lambda\right\}\right| \leq C_{1} e^{-C_{2} \lambda /\|\log v\|_{\text {Вмо }}}$.

By (33) and (34), we have

$$
\begin{aligned}
\mid\left\{w \in \partial D: \mid \log v \circ \phi_{z}(w)-\log (\right. & (z)) \mid>\lambda\} \mid \\
& \leq C_{1} e^{-C_{2} \lambda /\|\log v\|_{\text {вMO }}} .
\end{aligned}
$$

Let $E$ be a subset of $\partial D$. For $z \in D$, let

$$
\omega_{z}(E)=\int_{E} P(z, w) d w .
$$


Then (35) is equivalent to

(36) $\omega_{z}\{w \in \partial D:|\log v(w)-(\log v)(z)|>\lambda\} \mid \leq C_{1} e^{-C_{2} \lambda /\|\log v\|_{\text {BMO }}}$.

Let $E_{k}=\left\{w \in \partial D: e^{k} v(z)^{-1} \leq v(w)^{-1} \leq e^{k+1} v(z)^{-1}\right\}$. Then

$$
v^{-1 /(p-1)}(z) \leq v(z)^{-1 /(p-1)}\left(1+\sum_{k=0}^{\infty} \omega\left(E_{k}\right) e^{(k+1) /(p-1)}\right) .
$$

By (36), we have

$$
v(z)^{-1 /(p-1)}\left(1+C_{1} \sum_{k=0}^{\infty} e^{(k+1) /(p-1)-\left(C_{2} k\right) /\|\log v\|_{\mathrm{BMO}}}\right) \leq C v(z)^{-1 /(p-1)}
$$

if $1 /(p-1)<C_{2} /\|\log v\|_{\mathrm{BMO}}$. Therefore if $p>1+\|\log w\|_{\mathrm{BMO}} / C_{2}$, we have

$$
v(z)\left(v^{-(1 /(p-1))}(z)\right)^{p-1} \leq C,
$$

for all $z \in D$. This completes the proof of the theorem.

Now we show another characterization of invariant $A_{\infty}$ weights.

Theorem 8. A weight function $v$ is an invariant $A_{\infty}$ if and only if there is a constant $C>0$ such that

$$
\int_{D} P_{s}(z) v(z)\left(\frac{|\nabla v(z)|^{2}}{v(z)^{2}}\right)\left(1-|z|^{2}\right) d A(z) \leq C v(s)
$$

for all $s \in D$ where $P_{s}(z)=(1-|s|) /|1-\bar{s} z|^{2}$ for $s, z \in D$, and

$$
v(z) \cong \frac{1}{\left|I_{z}\right|} \int_{I_{z}} v(w) d w
$$

for all $z \in D$.

Proof. Suppose that $v$ is an invariant $A_{\infty}$ weight. By Cauchy and Jensen inequalities

$$
\exp ((\log v)(z)) \leq\left(v^{1 / 2}(z)\right)^{2} \leq v(z),
$$

and since $v$ is an invariant weight,

$$
v(z) \exp (-(\log v)(z)) \leq C .
$$


Therefore

$$
v(z) \leq C\left(v^{1 / 2}(z)\right)^{2} .
$$

In addition by Theorem $6,\left(|\nabla v(z)|^{2} / v(z)^{2}\right)\left(1-|z|^{2}\right)$ is a Carleson measure,

$$
\begin{aligned}
\int_{D} v\left(\frac{|\nabla v(z)|^{2}}{v(z)^{2}}\right)( & \left.-|z|^{2}\right) d A(z) \\
& \leq \int_{D}\left(\left(v^{1 / 2}\right)(z)\right)^{2}\left(\frac{|\nabla v(z)|^{2}}{v(z)^{2}}\right)\left(1-|z|^{2}\right) d A(z) \\
& \leq C \int_{\partial D}\left(v^{1 / 2}\left(e^{i \theta}\right)\right)^{2} d \theta \\
& =C \int_{\partial D} v\left(e^{i \theta}\right) d \theta \\
& =C v(0) .
\end{aligned}
$$

In the above inequality replacing $v$ by $v \circ \phi_{z}$ and making the change of variables give

$$
\int_{D} P_{s}(z) v(z)\left(\frac{|\nabla v(z)|^{2}}{v(z)^{2}}\right)\left(1-|z|^{2}\right) d A(z) \leq C v(s)
$$

for all $s \in D$.

Suppose that a weight function $v$ satisfies two conditions in Theorem 8. By Theorem 5, we need only to show that $v$ is an $A_{\infty}$ weight. By Green's formula, we have

$$
\begin{aligned}
v(z) \log (v(z))-(v & \log v)(z) \\
& =-\int_{D} \log \left|\frac{1-\bar{z} w}{z-w}\right| \Delta(v(w) \log (v(w))) d A(w) .
\end{aligned}
$$

It is easy to check that

$$
\Delta(v(w) \log (v(w)))=v(w)\left|\frac{|\nabla v(w)|^{2}}{v(w)^{2}}\right|
$$

So

$v(z) \log (v(z))-(v \log v)(z)=-\int_{D} \log \left|\frac{1-\bar{z} w}{z-w}\right| v(w)\left|\frac{|\nabla v(w)|^{2}}{v(w)^{2}}\right| d A(w)$. 
By the first condition in Theorem 8, we have

$$
\int P_{z}(w) v(w) \log \left(\frac{v(w)}{v(z)}\right) d w \leq C v(z)
$$

Hence

$$
\int P_{z}(w)\left(\frac{v(w)}{v(z)}\right)\left(\log \left(\frac{v(w)}{v(z)}\right)\right) d w \leq C .
$$

Now we write $\log x=\log ^{+} x-\log ^{-} x$. It is easy to see that $x \log ^{-} x \leq C$. Then

$$
\int P_{z}(w)\left(\frac{v(w)}{v(z)}\right)\left(\log ^{+}\left(\frac{v(w)}{v(z)}\right)\right) d w \leq C,
$$

for all $z \in D$.

For $L>10$, then we have

$$
\int_{\{v>L v(z)\}} P_{z}(w) v(w) d w \leq \frac{C}{\log L} v(z) .
$$

Hence

$$
\int_{\left\{w \in I_{z}: v>L v(z)\right\}} v(w) d w \leq \frac{C}{\log L} v(z)\left|I_{z}\right| .
$$

For any subset $E$ of $I_{z}$ with $|E| \leq\left|I_{z}\right| / L^{2}$,

$$
\begin{aligned}
\int_{E} v(w) d w & =\int_{\{E: v(w) \leq L v(z)\}} v(w) d w+\int_{\{E: v(w)>L v(z)\}} v(w) d w \\
& \leq L v(z)|E|+\frac{C}{\log L} v(z)\left|I_{z}\right|
\end{aligned}
$$

By the second condition in Theorem 8, we have

$$
\begin{aligned}
\int_{E} v(w) d w & \leq \frac{1}{L} \int_{I_{z}} v(w) d w+\frac{C}{\log L} \int_{I_{z}} v(w) d w \\
& =\left(\frac{1}{L}+\frac{C}{\log L}\right) \int_{I_{z}} v(w) d w
\end{aligned}
$$

If $L$ is sufficiently large, there are two numbers $0<\alpha=1 / L^{2}<1$ and $0<\beta=1 / L+C / \log L<1$ such that whenever $E \subset I_{z}$ and $|E| \leq \alpha\left|I_{z}\right|$,

$$
\int_{E} v(w) d w \leq \beta\left|I_{z}\right|
$$


We have proved that $v$ is an $A_{\infty}$ weight by [3]. This completes the proof of Theorem 8.

In [25], it is shown that if a measure $\mu$ in $D$ satisfies

$$
\int_{D} P_{s}(z) v(z)^{2} d \mu(z) \leq C v(s)
$$

then the following imbedding theorem

$$
\int_{D}\left|\left(f v^{1 / 2}\right)(z)\right|^{2} d \mu(z) \leq K(C)\|f\|_{2}^{2}
$$

for all $f \in L^{2}$, holds. We will characterize invariant $A_{\infty}$ weights by the so-called imbedding theorem.

Theorem 9. A weight function $v$ is an invariant $A_{\infty}$ weight if and only if there is a constant $C>0$ such that

$$
\begin{aligned}
\int_{D}\left|\left(f v_{s}\right)(z)\right|^{2} \frac{1}{v_{s}(z)}\left(\frac{\left|\nabla v_{s}(z)\right|^{2}}{v_{s}(z)^{2}}\right)(1- & \left.|z|^{2}\right) d A(z) \\
& \leq C \int_{\partial D}\left|f\left(e^{i \theta}\right)\right|^{2} v_{s}\left(e^{i \theta}\right) d \theta
\end{aligned}
$$

for all $f \in L^{2}\left(v_{s}\right)$ and all $s \in D$, where $v_{s}=v \circ \phi_{s}$, and

$$
v(z) \cong \frac{1}{\left|I_{z}\right|} \int_{I_{z}} v(w) d w
$$

for all $z \in D$.

Proof. Since the condition in $v$ is an invariant $A_{\infty}$, by Theorem 8 , we have that there is a constant $C>0$ such that

$$
\int_{D} P_{s}(z) v(z)\left(\frac{|\nabla v(z)|^{2}}{v(z)^{2}}\right)\left(1-|z|^{2}\right) d A(z) \leq C v(s),
$$

for all $s \in D$. The above condition is invariant. So we need only to show that

$$
\int_{D}|(f v)(z)|^{2} \frac{1}{v(z)}\left(\frac{|\nabla v(z)|^{2}}{v(z)^{2}}\right)\left(1-|z|^{2}\right) d A(z) \leq C \int_{\partial D}\left|f\left(e^{i \theta}\right)\right|^{2} v\left(e^{i \theta}\right) d \theta
$$


for all $f \in L^{2}(v)$. Let $g=f v^{1 / 2}$ and

$$
d \mu=\frac{\left(\frac{|\nabla v(z)|^{2}}{v(z)^{2}}\right)\left(1-|z|^{2}\right) d A(z)}{v} .
$$

Then it is sufficient to show

$$
\int_{D}\left|\left(g v^{1 / 2}\right)(z)\right|^{2} d \mu(z) \leq C \int_{\partial D}\left|g\left(e^{i \theta}\right)\right|^{2} d \theta .
$$

By Theorem 1.1 in [25], it is sufficient to show that

$$
\int_{D} P_{s}(z)(v(z))^{2} d \mu(z) \leq C v(s) .
$$

This is equivalent to

$$
\int_{D} P_{s}(z) v(z)\left(\frac{|\nabla v(z)|^{2}}{v(z)^{2}}\right)\left(1-|z|^{2}\right) d A(z) \leq C v(s) .
$$

Conversely suppose that $v$ satisfies the conditions in Theorem 9. If we let $f$ be 1 in the first condition we have

$$
\int_{D}\left|\left(v_{s}\right)(z)\right|^{2} \frac{1}{v_{s}(z)}\left(\frac{\left|\nabla v_{s}(z)\right|^{2}}{v_{s}(z)^{2}}\right)\left(1-|z|^{2}\right) d A(z) \leq C \int_{\partial D} v_{s}\left(e^{i \theta}\right) d \theta .
$$

Making the change of variable implies

$$
\int_{D} P_{s}(z) v(z)\left(\frac{|\nabla v(z)|^{2}}{v(z)^{2}}\right)\left(1-|z|^{2}\right) d A(z) \leq C v(s),
$$

for all $s \in D$ where $P_{s}(z)=\left(1-|s|^{2}\right) /|1-\bar{s} z|^{2}$ for $s, z \in D$. By Theorem 8 we conclude that $v$ is an invariant $A_{\infty}$ weight. This completes the proof of Theorem 9 .

\section{Toeplitz operators and Hankel operators.}

Let $f$ be in $L^{2}$. The Toeplitz operator $T_{f}$ and the Hankel operator $H_{f}$ with symbol $f$ are defined by $T_{f} p=P(f p)$, and $H_{f} p=(1-P)(f p)$, for all analytic polynomials $p$. Obviously they are densely defined on the Hardy space $H^{2}$. In this section we will show several sufficient 
conditions for the product of two Toeplitz operators or Hankel operators to be bounded.

Theorem 10. Let $X$ and $Y$ be two Banach function spaces such that $N_{X^{\prime}}$ and $N_{Y^{\prime}}$ map $L^{2}$ to $L^{2}$. Suppose that $(f, g)$ is a pair of functions in $L^{2}$ such that

$$
\sup _{z \in D}\left\|f_{-} \circ \phi_{z}-f_{-}(z)\right\|_{X}\left\|g_{-} \circ \phi_{z}-g_{-}(z)\right\|_{Y}<\infty .
$$

Then the product $H_{f}^{*} H_{g}$ is bounded on the Hardy space $H^{2}$.

Proof. Let $\phi$ and $\psi$ be in $H^{2}$. Then

$$
\left\langle H_{f}^{*} H_{g} \psi, \phi\right\rangle=\left\langle H_{g} \psi, H_{f} \phi\right\rangle .
$$

Using the Littlewood-Paley formula, we have

$$
\left\langle H_{f}^{*} H_{g} \psi, \phi\right\rangle=\iint\left\langle\nabla\left(H_{g} \psi\right)(z), \nabla\left(H_{f} \phi\right)(z)\right\rangle \log \frac{1}{|z|} d A(z) .
$$

Define

$$
\operatorname{Term}_{I}=\iint_{|z|>1 / 2}\left\langle\nabla\left(H_{g} \psi\right)(z), \nabla\left(H_{f} \phi\right)(z)\right\rangle \log \frac{1}{|z|} d A(z)
$$

and

$$
\operatorname{Term}_{I I}=\iint_{|z|<1 / 2}\left\langle\nabla\left(H_{g} \psi\right)(z), \nabla\left(H_{f} \phi\right)(z)\right\rangle \log \frac{1}{|z|} d A(z) .
$$

It is easy to verify that there is a compact operator $K$ on $H^{2}$ such that

$$
\operatorname{Term}_{I I}=\langle K \psi, \phi\rangle
$$

We claim that there is a constant $C>0$ such that

$$
\left|\operatorname{Term}_{I}\right| \leq C \sup _{z \in D}\left\|f_{-} \circ \phi_{z}-f_{-}(z)\right\|_{X}\left\|g_{-} \circ \phi_{z}-g_{-}(z)\right\|_{Y}\|\psi\|_{2}\|\phi\|_{2}
$$

So

$$
\left\|H_{f}^{*} H_{g}\right\| \leq\|K\|+C \sup _{z \in D}\left\|f_{+} \circ \phi_{z}-f_{+}(z)\right\|_{X}\left\|g_{-} \circ \phi_{z}-g_{-}(z)\right\|_{Y}
$$


Now we turn to the proof of the claim. Fix an $a>0$ for which the distribution function inequality (22) holds. For $w \in \partial D$, let $\rho(w)$ denote the maximum of those numbers $\varepsilon$ for which

$$
\begin{aligned}
& A_{\varepsilon}\left(H_{f} \phi\right)(w) A_{\varepsilon}\left(H_{g} \psi\right)(w) \\
\leq & a\left\|f_{-} \circ \phi_{z}-f_{-}(z)\right\|_{X}\left\|g_{-} \circ \phi_{z}-g_{-}(z)\right\|_{Y} N_{X^{\prime}}(\phi)(w) N_{Y^{\prime}}(\psi)(w) .
\end{aligned}
$$

Thus

$$
\begin{aligned}
\int_{\partial D} & A_{\rho(w)}\left(H_{f} \phi\right)(w) A_{\rho(w)}\left(H_{g} \psi\right)(w) d w \\
\leq & a \sup _{z \in D}\left\|f_{-} \circ \phi_{z}-f_{-}(z)\right\|_{X}\left\|g_{-} \circ \phi_{z}-g_{-}(z)\right\|_{Y} \\
& \cdot \int_{\partial D} N_{X^{\prime}}(\phi)(w) N_{Y^{\prime}}(\psi)(w) d w \\
\leq & a \sup _{z \in D}\left\|f_{-} \circ \phi_{z}-f_{-}(z)\right\|_{X}\left\|g_{-} \circ \phi_{z}-g_{-}(z)\right\|_{Y} \\
& \cdot\left\|N_{X^{\prime}}(\phi)\right\|_{2}\left\|N_{Y^{\prime}}(\psi)\right\|_{2} \\
\leq & a \sup _{z \in D}\left\|f_{-} \circ \phi_{z}-f_{-}(z)\right\|_{X}\left\|g_{-} \circ \phi_{z}-g_{-}(z)\right\|_{Y}\|\phi\|_{2}\|\psi\|_{2} .
\end{aligned}
$$

The last inequality holds because $N_{X^{\prime}}$ and $N_{Y^{\prime}}$ are bounded on $L^{2}$.

On the other hand, letting $\chi_{w}(z)$ denote the characteristic function of $\Gamma_{w, \rho(w)}$, we have

$$
\begin{aligned}
\int_{\partial D} A_{\rho(w)}\left(H_{f} \phi\right)(w) A_{\rho(w)}\left(H_{g} \psi\right)(w) d w \\
=\int_{\partial D}\left(\int_{\Gamma_{w, \rho(w)}}\left|\nabla\left(H_{f} \phi\right)(z)\right|^{2} d A(z)\right)^{1 / 2} \\
\\
\quad\left(\int_{\Gamma_{w, \rho(w)}}\left|\nabla\left(H_{g} \psi\right)(z)\right|^{2} d A(z)\right)^{1 / 2} d \omega \\
\geq \int_{|z|>1 / 2} \int_{\partial D} \chi_{w}(z)\left|\nabla\left(H_{f} \phi\right)(z)\right|\left|\nabla\left(H_{g} \psi\right)(z)\right| d w d A(z) .
\end{aligned}
$$

Now the distribution function inequality (22) tells us that $\rho(w) \geq 2(1-$ $|z|)$ on a subset of $I_{z}$ whose measure is at least $C_{a}(1-|z|)$. If $w \in I_{z}$ and $\rho(w) \geq 2(1-|z|)$, then $z$ in $\Gamma_{w, \rho(w)}$. Thus $\chi_{w}(z)=1$ on a subset 
of $I_{z}$ of measure at least $C_{a}(1-|z|)$. Combining this observation with the previous inequality, we obtain

$$
\begin{aligned}
\int_{\partial D} A_{\rho(w)} & \left(H_{f} \phi\right)(w) A_{\rho(w)}\left(H_{g} \psi\right)(w) d w \\
& \geq C_{a} \int_{|z|>1 / 2}\left|\nabla\left(H_{f} \phi\right)(z)\right|\left|\nabla\left(H_{g} \psi\right)(z)\right|(1-|z|) d A(z) \\
& \geq C_{a}\left|\operatorname{Term}_{I}\right|
\end{aligned}
$$

So

$$
\left|\operatorname{Term}_{I}\right| \leq C \sup _{z \in D}\left\|f_{-} \circ \phi_{z}-f_{-}(z)\right\|_{X}\left\|g_{-} \circ \phi_{z}-g_{-}(z)\right\|\left\|_{Y}\right\| \phi\left\|_{2}\right\| \psi \|_{2} .
$$

This completes the proof of the theorem.

Theorem 11. Let $X$ and $Y$ be two Banach function spaces such that $N_{X^{\prime}}$ and $N_{Y^{\prime}}$ map $L^{2}$ to $L^{2}$. Suppose that $(f, g)$ is a pair of outer functions in $H^{2}$ such that

$$
\sup _{z \in D}\left\|f \circ \phi_{z}\right\|_{X}\left\|g \circ \phi_{z}\right\|_{Y}<\infty .
$$

Then the product $T_{f} T_{\bar{g}}$ is bounded on the Hardy space $H^{2}$.

Proof. Let $\phi$ and $\psi$ be two polynomials.

$$
\left\langle T_{f} T_{\bar{g}} \psi, \phi\right\rangle\left\langle T_{\bar{g}} \psi, T_{\bar{f}} \phi\right\rangle .
$$

Using the Littlewood-Paley formula, we have

$$
\left\langle T_{\bar{g}} \psi, T_{\bar{f}} \phi\right\rangle=\int_{D}\left\langle\nabla\left(T_{\bar{g}} \psi\right)(z), \nabla\left(T_{\bar{g}} \phi\right)(z)\right\rangle \log \frac{1}{|z|} d A(z) .
$$

Define

$$
\operatorname{Term}_{I}=\iint_{|z|>1 / 2}\left\langle\nabla\left(T_{\bar{g}} \psi\right)(z), \nabla\left(T_{\bar{f}} \phi\right)(z)\right\rangle \log \frac{1}{|z|} d A(z) .
$$

and

$$
\operatorname{Term}_{I I}=\iint_{|z|<1 / 2}\left\langle\nabla\left(T_{\bar{g}} \psi\right)(z), \nabla\left(T_{\bar{f}} \phi\right)(z)\right\rangle \log \frac{1}{|z|} d A(z)
$$


It is easy to verify that there are constants $C_{1}>0$ and $C_{2}>0$ such that

$$
\left|\operatorname{Term}_{I I}\right| \leq C_{1}\|\psi\|_{2}\|\phi\|_{2}
$$

and

$$
\left|\operatorname{Term}_{I I}\right| \leq C_{2}\|\psi\|_{2}\|\phi\|_{2}
$$

We will show that there are the same estimates of Term ${ }_{I}$ as above ones.

Let $X$ and $Y$ be two Orlicz spaces. Fix an $a>0$ for which the distribution function inequality (21) holds. For $w \in \partial D$, let $\rho(w)$ denote the maximum of those numbers $\varepsilon$ for which

$$
\begin{aligned}
A_{\varepsilon}\left(T_{\bar{f}} \phi\right)(w) A_{\varepsilon}\left(T_{\bar{g}} \psi\right)(w) & \\
& \leq a\left\|f \circ \phi_{z}\right\|_{X}\left\|g \circ \phi_{z}\right\|_{Y} N_{X^{\prime}}(\phi)(w) N_{Y^{\prime}}(\psi)(w) .
\end{aligned}
$$

Thus

$$
\begin{aligned}
\int_{\partial D} & A_{\rho(w)}\left(T_{\bar{f}} \phi\right)(w) A_{\rho(w)}\left(T_{\bar{g}} \psi\right)(w) d w \\
& \leq a \sup _{z \in D}\left\|f \circ \phi_{z}\right\|_{X}\left\|g \circ \phi_{z}\right\|_{Y} \int_{\partial D} N_{X^{\prime}}(\phi)(w) N_{Y^{\prime}}(\psi)(w) d w .
\end{aligned}
$$

On the other hand, letting $\chi_{w}(z)$ denote the characteristic function of $\Gamma_{w, \rho(w)}$, we have

$$
\begin{aligned}
\int_{\partial D} A_{\rho(w)}\left(T_{\bar{f}} \phi\right)(w) A_{\rho(w)}\left(T_{\bar{g}} \psi\right)(w) d w \\
=\int_{\partial D}\left(\int_{\Gamma_{w, \rho(w)}}\left|\nabla\left(T_{\bar{f}} \phi\right)(z)\right|^{2} d A(z)\right)^{1 / 2} \\
\quad \cdot\left(\int_{\Gamma_{w, \rho(w)}}\left|\nabla\left(T_{\bar{g}} \psi\right)(z)\right|^{2} d A(z)\right)^{1 / 2} d \omega \\
\geq \int_{|z|>1 / 2} \int_{\partial D} \chi_{w}(z)\left|\nabla\left(T_{\bar{f}} \phi\right)(z)\right|\left|\nabla\left(T_{\bar{g}} \psi\right)(z)\right| d w d A(z) .
\end{aligned}
$$

Now the distribution function inequality (21) tells us that $\rho(w) \geq 2(1-$ $|z|)$ on a subset of $I_{z}$ whose measure is at least $C_{a}(1-|z|)$. If $w \in I_{z}$ and $\rho(w) \geq 2(1-|z|)$, then $z$ in $\Gamma_{w, \rho(w)}$. Thus $\chi_{w}(z)=1$ on a subset of $I_{z}$ of measure at least $C_{a}(1-|z|)$. Combining this observation with 
the previous inequality, we obtain

$$
\begin{aligned}
\int_{\partial D} A_{\rho(w)} & \left(T_{\bar{f}} \phi\right)(w) A_{\rho(w)}\left(T_{\bar{g}} \psi\right)(w) d w \\
& \geq C_{a} \int_{|z|>1 / 2}\left|\nabla\left(T_{\bar{f}} \phi\right)(z)\right|\left|\nabla\left(T_{\bar{g}} \psi\right)(z)\right|(1-|z|) d A(z) \\
& \geq C_{a}\left|\operatorname{Term}_{I}\right| .
\end{aligned}
$$

So

$$
\begin{aligned}
\left|\operatorname{Term}_{I}\right| & \leq a \sup _{z \in D}\left\|f \circ \phi_{z}\right\|_{X}\left\|g \circ \phi_{z}\right\|_{Y} \int N_{X^{\prime}}(\phi)(w) N_{Y^{\prime}}(\psi)(w) d w \\
& \leq a \sup _{z \in D}\left\|f \circ \phi_{z}\right\|_{X}\left\|g \circ \phi_{z}\right\|_{Y}\left\|N_{X^{\prime}}(\phi)\right\|_{2}\left\|N_{Y^{\prime}}(\psi)\right\|_{2} \cdot
\end{aligned}
$$

Since $N_{X^{\prime}}$ and $N_{Y^{\prime}}$ are bounded on $L^{2}$, we have

$$
\left|\operatorname{Term}_{I}\right| \leq a \sup _{z \in D}\left\|f \circ \phi_{z}\right\|_{X}\left\|g \circ \phi_{z}\right\|_{Y}\|\phi\|_{2}\|\psi\|_{2}
$$

This completes the proof of the theorem.

Acknowledgements. We thank the referee for useful comments.

\section{References.}

[1] Bennett, C., Sharpley, R., Interpolation of operators. Academic Press, 1988.

[2] Buckley, S., Estimates for operator norms on weighted spaces and reverse Jensen inequalities. Trans. Amer. Math. Soc. 340 (1993), 253-272.

[3] Coifman, R. R., Fefferman, C., Weighted norm inequalities for maximal functions and singular integrals. Studia Math. 51 (1974), 241-250.

[4] Cotlar, M., Sadosky, C., On the Helson-Szegö theorem and a related class of modified Toeplitz kernels, Harmonic analysis in Eucliden spaces. Proc. Symp. Pure Math. 35 (1979), 383-407.

[5] Cotlar, M., Sadosky, C., On some $L^{p}$ versions of the Helson-Szegö theorem, Conference on harmonic analysis in Honor of Antoni Zygmund, (1983), 306-317. 
[6] Cruz-Uribe, SFO D., The invertibility of the product of unbounded Toeplitz operators. Integral equations and operator theory 20 (1994), 231-237.

[7] Fefferman, R., Kenig, C. F., Pipher, J., The theory of weights and the Dirichlet problem for elliptic equations. Ann of Math. 134 (1991), 65124.

[8] Fujii, N., A condition for a two weight norm inequality for singular integral operators. Studia Math. 98 (1991), 175-190.

[9] Garcia-Cuerva, J., Rubio de Francia, J. L., Weighted norm inequalities and related topics. North-Holland, 1985.

[10] Garnett, J. B., Bounded analytic functions. Pure and Applied Math. 96 Academic Press, 1981.

[11] Hruscev, S. V., A description of weights satisfying the $A_{\infty}$ condition of Muckenhoupt. Proc. Amer. Math. Soc. 90 (1984), 253-257.

[12] Hunt, R. A., Muckenhoupt, B., Wheeden, R. L., Weighted norm inequalities for the conjugate function and Hilbert transform. Trans. Amer. Math. Soc. 176 (1973), 227-251.

[13] Krasnosel'skii, M., Rutickii, J., Convex functions and Orlicz spaces. Noordhoff, 1961.

[14] Muckenhoupt, B., Weighted norm inequalities for the Hardy maximal function, Trans. Amer. Math. Soc. 165 (1972), 207-226.

[15] Muckenhoupt, B., Weighted norm inequalities for classical operators. Proc. Symp. Pure Math. 35 (1979), 69-83.

[16] Muckenhoupt, B., Wheeden, R., Two weight function norms inequality for the Hardy-Littlewood maximal function and the Hilbert transform. Studia Math. 55 (1976), 279-294.

[17] Neugebauer, C., Inserting $A_{p}$-weights. Proc. Amer. Math. Soc. 87 (1983), 644-648.

[18] Rao, M., Ren, Z., Theory of Orlicz space. Marcel Dekker, Inc., 1991.

[19] Pérez, C., On sufficient conditions for the boundedness of the HardyLittlewood maximal operator between weighted $L^{p}$-spaces with different weights. Proc. London Math. Soc. 71 (1995), 135-157.

[20] Sarason, D., Products of Toeplitz operators. In: Linear and Complex Analysis Problem, Book 3, Part I. Ed. V.P. Havin and N.K. Nikolskii. Lecture Notes Math. 1573 (1994), 318-319, Springer-Verlag.

[21] Sawyer, E. T., A characterization of a two-weight norm inequality for maximal operators. Studia Math. 75 (1982), 1-11.

[22] Sawyer, E. T., Norm inequalities relating singular integrals and the maximal function. Studia Math. 76 (1983), 253-263. 
[23] Sawyer, E. T., Wheeden, R. L., Weighted inequalities for fractional integrals on Euclidean and homogeneous spaces. Amer. J. Math. 114 (1992), 813-874.

[24] Strömberg, J. -O., Non-equivalence between two kinds of conditions on weight functions. Harmonic analysis in Eucliden spaces. Proc. Symp. Pure Math. 35 (1979), 141-148.

[25] Treil, S., Volberg, A., A simple proof of the Hunt-Muckenhoupt-Wheeden theorem. Algebra i Analiz 7 (1995), 205-226.

[26] Wolff, T., Counterexamples to two variants of the Helson-Szegö theorem. Preprint (1983).

[27] Zheng, D., The distribution function inequality and products of Toeplitz operators and Hankel operators. J. Funct. Anal. 138 (1996), 477-501.

Recibido: 3 de noviembre de 1.995

Revisado: 30 de abril de 1.996

Sergei Treil, Alexander Volberg

Department of Mathematics Michigan State University East Lansing, MI 48824

U.S.A.

treil@math.msu.edu

volberg@math.msu.edu

and

Dechao Zheng

Department of Mathematics

Vanderbilt University

Nashville, TN 37240

U.S.A.

zheng@math . vanderbilt.edu 